\title{
ESTUDIO TAXONÓMICO Y BIOMÉTRICO DE Badulesia tenera (HARTT IN DAWSON, 1868), TRILOBITA. CAESARAUGUSTIENSE (CÁMBRICO MEDIO) DE LAS CADENAS IBÉRICAS (NE DE ESPAÑA)
}

\author{
Juan B. CHIRIVELLA MARTORELL', \\ Rodolfo GOZALO I Eladio LIÑÁN $^{2}$ \\ ' Departamento de Geología. Universitat de València. C/ Dr. Moliner 50. E- \\ 46100-Burjassot (Valencia) ESPAÑA. Correo Electrónico: \\ Rodolfo.Gozalo@uv.es \\ 2 Departamento de Ciencias de la Tierra. Universidad de Zaragoza. E-50009- \\ Zaragoza ESPAÑA.
}

Chirivella Martorell, J.B., Gozalo, R. y Liñán, E. 2003. Estudio taxonómico y biométrico de Badulesia tenera (Hartt in Dawson, 1868), Trilobita. Caesaragustiense (Cámbrico Medio) de las Cadenas Ibéricas (NE de España). [Taxonomic and biometric study of Badulesia tenera (Hartt in Dawson, 1868), Trilobita. Caesaraugustan (Middle Cambrian) from the Iberian Chains (NE Spain).] Revista Española de Paleontología, 18(1), 83-101. ISSN 0213-6937.

\begin{abstract}
ABSTRAC'T
In Jarque 1 section (Iberian Chains) a stratigraphic interval of $18 \mathrm{~m}$ comprising upper parts of the Mansilla Formation and lower parts of the overlying Murero Formation has yielded an almost continuous record of Badulesia tenera (Hartt in Dawson, 1868), which includes more than 200 specimens. This species is very abundant in some levels. A morphological and morphometric analysis of the recorded populations allow us to emend the diagnosis of the genus Badulesia Sdzuy, 1968 and of the species Badulesia tenera. In addition, the observed variations have led to the description of two cranidial morphotypes: the Morphotype A without preglabellar field and the Morphotype B with preglabellar field. Widely varying rank of cephalon characters have also been analysed and are thought to be related to intraspecific variations.

The cranidial bivariate analysis confirms that the great part of the variations results from growth. The analysis shows a continuous variation within the samples. Therefore, we can consider that they are indicative of intraspecific variations. Only when one of the dimensions being compared is the cranidial border length (Lb) or the preglabellar area length (Lap), it is possible to observe two different distributions of points. Every group is composed of specimens of only one of the morphotypes. Also, in these cases, the Pearson correlation and the determination $\left(\mathrm{R}^{2}\right)$ coefficients are lower than when the comparison is made between other dimensions. This indicates a lower control over growth for these dimensions, and high variability patterns. Another fact observed is that cranidial length versus width consistently shows lower Pearson and determination coefficients than either length versus length or width versus width comparisons.

Furthermore, this biometric study proves that the taxonomic determinations are also possible for distorted material preserved either as internal or external moulds. Those determinations are as reliable as determinations for non-distorted materials. The only condition is that the number of specimens has to be sufficient to allow a population study.

From a biostratigraphic point of view the relevance of the first appearance datum (FAD) of Badulesia tenera is clear. This FAD is currently considered to be the marker of the beginning of the Caesaraugustan Stage, which allows a good correlation between the Avalonian and Mediterranean subprovinces.
\end{abstract}

Keywords: Badulesia tenera (Trilobita), morphotypes, Caesaraugustan, Middle Cambrian, Iberian Chains, Spain.

\section{RESUMEN}

Se realiza el estudio de una sucesión estratigráfica desde la parte superior de la Formación Mansilla hasta la base de la Formación Murero, en la sección Jarque 1 de las Cadenas Ibéricas. En ella se ha encontrado un registro casi 
continuo de la especie Badulesia tenera (Hartt in Dawson, 1868), representada por unos doscientos especímenes, a lo largo de $18 \mathrm{~m}$, siendo muy abundante en algunos de los niveles estudiados. Todo ello ha permitido realizar un estudio morfológico y un análisis morfométrico de las poblaciones halladas. Esta revisión ha llevado a enmendar la diagnosis del género Badulesia Sdzuy, 1968 y de la especie estudiada. También se han definido dos morfotipos del cranidio de la especie Badulesia tenera: Morfotipo A sin área preglabelar y Morfotipo B con área preglabelar. Además, se analiza un amplio rango de variaciones en algunos caracteres del cefalón, que también se han considerado como variaciones intraespecíficas.

El análisis bivariante del cranidio ha permitido constatar que la mayoría de las variaciones biométricas están controladas por el crecimiento, mostrando un conjunto continuo que posibilita considerarlas como variaciones intraespecíficas. Solamente cuando se compara la longitud del borde del cranidio (Lb), o la longitud del área preglabelar (Lap), con la longitud del cranidio (Lc) se observa una clara separación entre las nubes de puntos correspondientes a ambos morfotipos; en estos casos los coeficientes de correlación de Pearson y de determinación $\left(\mathrm{R}^{2}\right)$ son más bajos que cuando se comparan otras dimensiones, lo que indica un menor control de crecimiento y una mayor variabilidad para estas dimensiones. También se ha constatado que cuando se comparan dimensiones medidas en direcciones perpendiculares, sus coeficientes de correlación son más bajos que cuando las dimensiones se miden en la misma dirección.

Además, el estudio biométrico demuestra que son posibles las determinaciones taxonómicas con material conservado en forma de moldes, tanto internos como externos, así como con material deformado y no deformado, y son igual de fiables. La única condición es que el número de ejemplares permita realizar un estudio poblacional.

Desde el punto de vista bioestratigráfico se confirma la importancia de la primera aparición de esta especie, considerada como el inicio del piso Caesaraugustiense que, además, permite constatar la bondad de las correlaciones establecidas entre las subprovincias Avalónica y Mediterránea, gracias a esta especie.

\section{Palabras clave: Badulesia tenera (Trilobita), morfotipos, Caesaraugustiense, Cámbrico Medio, Cadenas Ibéricas, España.}

\section{INTRODUCCIÓN}

La especie Badulesia tenera (Hartt in Dawson, 1868) se encuentra en numerosas localidades del Cámbrico Medio de las subprovincias Avalónica y Mediterránea; este hecho y su breve distribución estratigráfica le confieren una gran relevancia como elemento de correlación entre estas subprovincias, por lo que es necesario disponer de un buen conocimiento de su variabilidad morfológica. España es, por otra parte, el país con más citas de Badulesia tenera. Fue citada por primera vez por Sdzuy $(1968,1969)$, primero en varias localidades de la Cordillera Cantábrica y luego de las Cadenas Ibéricas, donde se ha encontrado en varias secciones. También fue hallada posteriormente en la Sierra de Córdoba por Liñán (1978).

Hasta el momento, no se había abordado un estudio morfológico de las poblaciones de esta especie, por lo que algunas de las variaciones que se habían observado fueron asignadas a la misma en nomenclatura abierta. Además, Sdzuy (1968) comenta la existencia de un amplio rango de variaciones morfológicas en el cranidio de esta especie, poniendo en evidencia la necesidad de hacer un análisis detallado de las mismas.

Hasta ahora, los hallazgos de esta especie en cada sección solían reducirse a un nivel con pocos especímenes, lo que posibilitaba la datación del nivel, pero no permitía un estudio de su variabilidad morfológica. El muestreo continuado en varias localidades de las Cadenas Ibéricas ha permitido incrementar el número de ejemplares y niveles donde se registra esta especie; de todas ellas, destaca la sección
Jarque 1, la cual presenta una secuencia continua de 18 $\mathrm{m}$ de espesor en los que se han encontrado 14 niveles que contienen Badulesia tenera, siendo ésta muy abundante en algunos de ellos. Ello ha permitido constatar la presencia de dos morfotipos cranidiales, uno de los cuales se había asignado previamente a esta especie con dudas.

Finalmente, como la primera aparición de Badulesia tenera marca el inicio del Piso Caesaraugustiense, propuesto por Liñán et al. (1993) para la subprovincia Mediterránea, de acuerdo con la modificación de Sdzuy et al. (1996), este evento consituye una de las mejores herramientas de correlación entre las subprovincias Mediterránea y Avalónica donde se encuentra bien representada esta especie. Por otro lado, las asociaciones de trilobites registradas junto con esta especie en las diferentes regiones posibilitan una buena correlación global de la Zona de Badulesia tenera tal y como señalan Sdzuy et al. (1999).

\section{SITUACIÓN GEOLÓGICA Y ESTRATIGRÁFICA DE LAS SECCIONES ESTUDIADAS}

Las secciones de las Cadenas Ibéricas de las que se figura material en este trabajo son tres: Jarque 1 (J1), Villafeliche 1 (Vi1) y Rambla de Valdemiedes 2 (RV2); la primera se ubica en la Cadena Ibérica Oriental y las otras dos en la Cadena Ibérica Occidental (Fig. 1). Desde un punto de vista geológico, las tres pertenecen a la Unidad de Badules de acuerdo con la delimitación de Gozalo y Liñán 


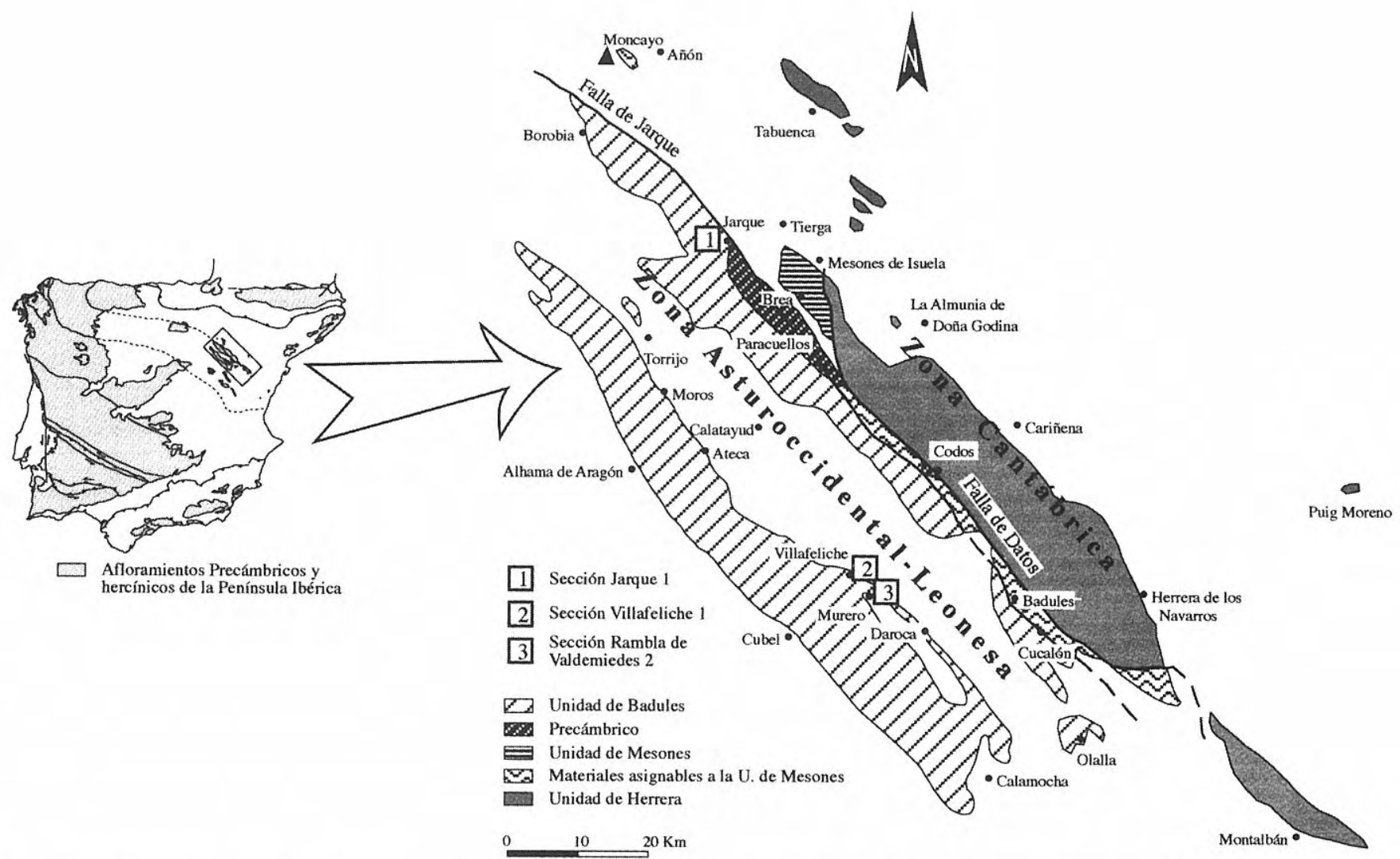

Figura 1. Situación geológica de los yacimientos de las Cadenas Ibéricas estudiados en este trabajo (modificado de Gozalo y Liñán, 1988).

Geological setting of the studied sections in the Iberian Chains (modified from Gozalo and Liñan, 1988).

(1988), en la que afloran materiales que van desde el Precámbrico Superior hasta el Ordovícico.

Estas secciones completas han sido descritas previamente; así, la sección RV2 ha sido estudiada por Liñán y Gozalo (1986), la sección Vil por Gozalo et al. (1993, 1996), y la sección J1 por Liñán et al. (1996). De estos trabajos se desprende que Badulesia tenera se registra desde los niveles superiores de la Formación Mansilla hasta la base de la Formación Murero. En la figura 2 se representa el intervalo de la sección Jarque 1 donde se han encontrado ejemplares de esta especie, así como el número de ejemplares recolectados en cada nivel.

Además del material aragonés, se ha figurado y medido material recogido por los autores en las cercanías de la ciudad de Saint John (New Brunswick), que es el área tipo de la especie, y en Terranova oriental, ambas en Cánada.

\section{TAXONOMÍA}

En este apartado se utiliza la nomenclatura propuesta por Sdzuy (1968) para este género, y la de Liñán y Gozalo (1986). El material figurado y/o medido se encuentra depositado en el Museo de Paleontología de la Universidad de Zaragoza con las siglas MPZ2001/131 a MPZ2001/199.
Superfamilia Solenopleuroidea Angelin, 1854

Familia Solenopleuridae Angelin, 1854

Subfamilia Solenopleuropsinae Thoral, 1947 Género Badulesia Sdzuy, 1968

Especie tipo: Liostracus granieri Thoral, 1935, por designación original, p. 111.

Diagnosis enmendada: Género de la Subfamilia Solenopleuropsinae cuya ornamentación del cefalón está compuesta por crestas. El cefalón posee un borde anterior y lateral a modo de cresta submarginal que se interrumpe en el ángulo genal. La glabela puede carecer de crestas o presentar cuatro pares de crestas coincidiendo con los lóbulos glabelares. La fixígena tiene los lobulos palpebrales y aristas oculares unidos, formando un par simétrico de estrechas crestas externas y, al menos, otro par de crestas interiores en cada una de las áreas palpebrales y postoculares, que delimitan éstas del surco axial de la glabela y del surco posterior del cranidio. El surco anterolateral del cefalón es ancho y profundo.

Genus of the Subfamily Solenopleuropsinae with cephalon ornamentation composed of ridges. The anterior and lateral cephalon borders look like a submarginal ridge cut in the genal angle. Glabella lacking ridges or bearing until four ridge pairs, which coindice with the glabellar lobes. Fixigena has the palpebral lobe and eyes ridge fused, making a pair of 


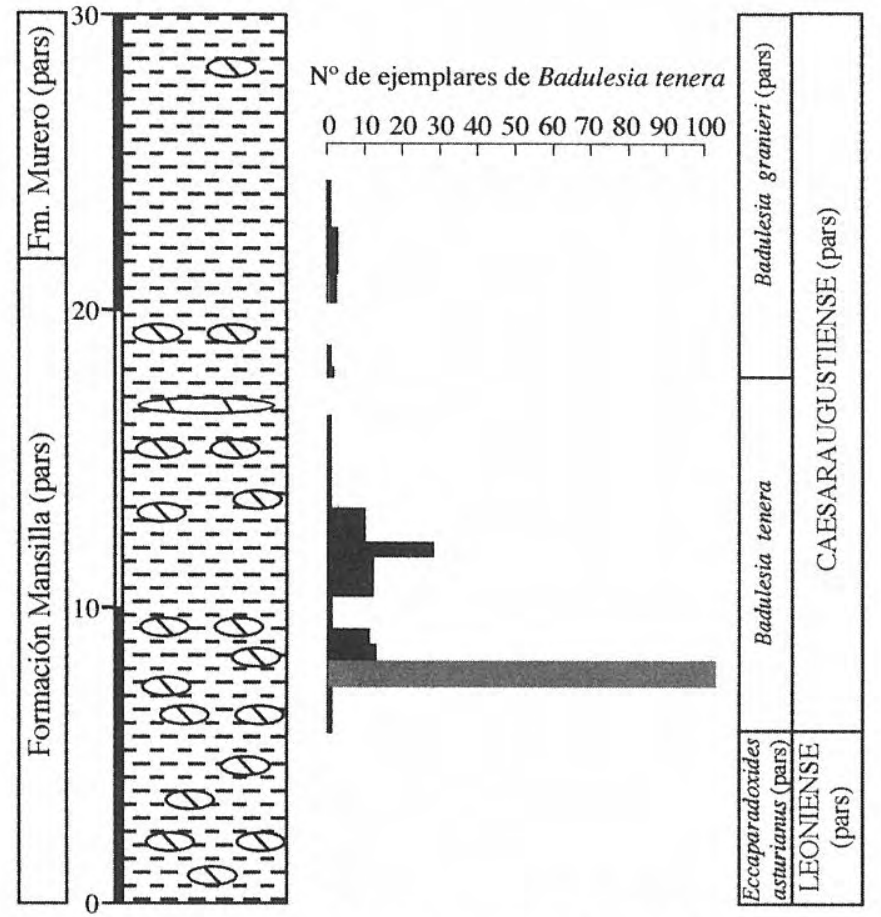

Figura 2. Niveles donde se ha encontrado Badulesia tenera, con indicación del número de ejemplares en cada nivel, en la sección Jarque 1 (J1). La trama de puntos señala el nivel J1/156.

Levels with Badulesia tenera in the Jarque section $1(J 1)$, with indication the number of specimens in every level. The point plot marks the level J1/156.

narrow outer ridges and, at least, another inner pair of ridges in the palpebral and postocular areas, which bounded these of the glabellar axial and posterior furrows. The anterolateral furrow of the cephalon is wide and deep.
Especies y subespecies: Además de la especie tipo: Conocephalites tener Hartt in Dawson, 1868, Pardailhania paschi Sdzuy, 1958, Badulesia juliverti juliverti Sdzuy, 1968 y Badulesia juliverti iugum Sdzuy, 1968.

\section{Discusión}

La incorporación a la diagnosis original, Sdzuy (1968: 111), de la referencia al borde anterior y lateral del cefalón en forma de cresta elevada, así como la necesidad de la presencia de al menos un par de crestas interiores en la fixígena, además de las crestas formadas por la unión de los lóbulos palpebrales y las aristas oculares, permiten diferenciar mejor el género Badulesia del resto de los géneros de Solenopleuropsinae.

El género Braintreella Wheeler, 1942 ha sido referido como un sinónimo más antiguo de Badulesia. Así, Bengtson y Fletcher (1983: 533) citan la especie Conocephalites tener como Braintreella tenera; esta propuesta es reflejada por Dean in Martin y Dean (1988: 21), quien haciendo referencia a la tesis doctoral inédita del Dr. Fletcher, dice: "Evidence for considering Badulesia a junior subjective synonym of Braintreella is not yet published, and for present purposes Badulesia is retained provisionally". Recientemente, Geyer y Landing (2001: 129-131) revisan el género Braintreella y su especie tipo, Ptychoparia rogersi Walcott, 1884; en esta revisión mantienen únicamente la especie tipo dentro del género Braintreella y comentan la posibilidad de que este género, a su vez, fuera un sinónimo subjetivo más moderno de algún otro género de Solenopleuridae, aunque la mala conservación del material de Braintreella rogersi les impide tomar una decisión. La nueva figuración y descripción de esta especie permite destacar algunos elementos morfológicos que separan nítidamente Badulesia de Braintreella, como son la presencia de un

Figura 3. Badulesia tenera (Hartt in Dawson, 1868). a: cranidio y segmento torácico, morfotipo A, molde interno (MPZ2001/159), J1/156 (x5). b: cranidio, morfotipo A, molde de látex (MPZ2001/138), J1/156 (x8). c: cranidio, morfotipo A, molde interno (MPZ2001/173), J1/158 (x8). d: cranidio, morfotipo A, molde interno (MPZ2001/163), J1/156 (x5). e: cranidio, morfotipo B, molde interno (MPZ2001/136), J1/156 (x5). f: cranidio, morfotipo A, molde interno (MPZ2001/174), J1/158 (x8). g: cefalón y tórax con 14 segmentos, morfotipo B, molde interno (MPZ2001/175), J1/158 (x5). h: cranidio, morfotipo A, molde interno (MPZ2001/166), J1/156 (x5). i: cranidio, morfotipo B, molde interno (MPZ2001/182), J1/160 (x5). j: cranidio y 6 segmentos torácicos, morfotipo B, molde de látex (MPZ2001/192), RV2/13.1 (x5). k: cranidio, morfotipo A, molde de látex (MPZ2001/198), J1/164 (x8). I: exuvio, cefalón y 8 segmentos torácicos, morfotipo A, molde de látex (MPZ2001/152), J1/156 (x4). m: cranidio, morfotipo B, molde interno (MPZ2001/191), RV2/13.1 (x8). n: cranidio, morfotipo B, molde interno (MPZ2001/140), J1/156 (x8).

$\boldsymbol{a}$ : cranidium and toracic segment, morphotype A, internal mould (MPZ2001/159), J1/156 (x5). b: cranidium, morphotype A, latex cast (MPZ2001/138), J1/156 (x8). c: cranidium, morphotype A, internal mould (MPZ2001/173), J1/158 (x8). d: cranidium, morphotype A, internal mould (MPZ2001/163), J1/156 (x5). e : cranidium, morphotype B, internal mould (MPZ2001/136), J1/156 (x5). $f$ : cranidium, morphotype A, internal mould (MPZ2001/174), J1/158 (x8). g: cephalon and thorax with 14 segments, morphotype B, internal mould (MPZ2001/175), J1/158 (x5). h: cranidium, morphotype A, internal mould (MPZ2001/166), J1/156 (x5). $\boldsymbol{i}$ : cranidium, morphotype B, internal mould (MPZ2001/182), J1/160 (x5). $j$ : cranidium and 6 thoracic segments, morphotype B, latex cast (MPZ2001/192), RV2/13.1 (x5). $\mathrm{k}$ : cranidium, morphotype A, latex cast (MPZ2001/198), $J 1 / 164$ (x8). $l$ : exuvia, cephalon and 8 thoracic segments, morphotype A, latex cast (MPZ2001/152), J1/156 (x4). m: cranidium, morphotype B, internal mould (MPZ2001/191), RV2/13.1 (x8). $n$ : cranidium, morphotype B, internal mould (MPZ2001/140), J1/156 (x8). 

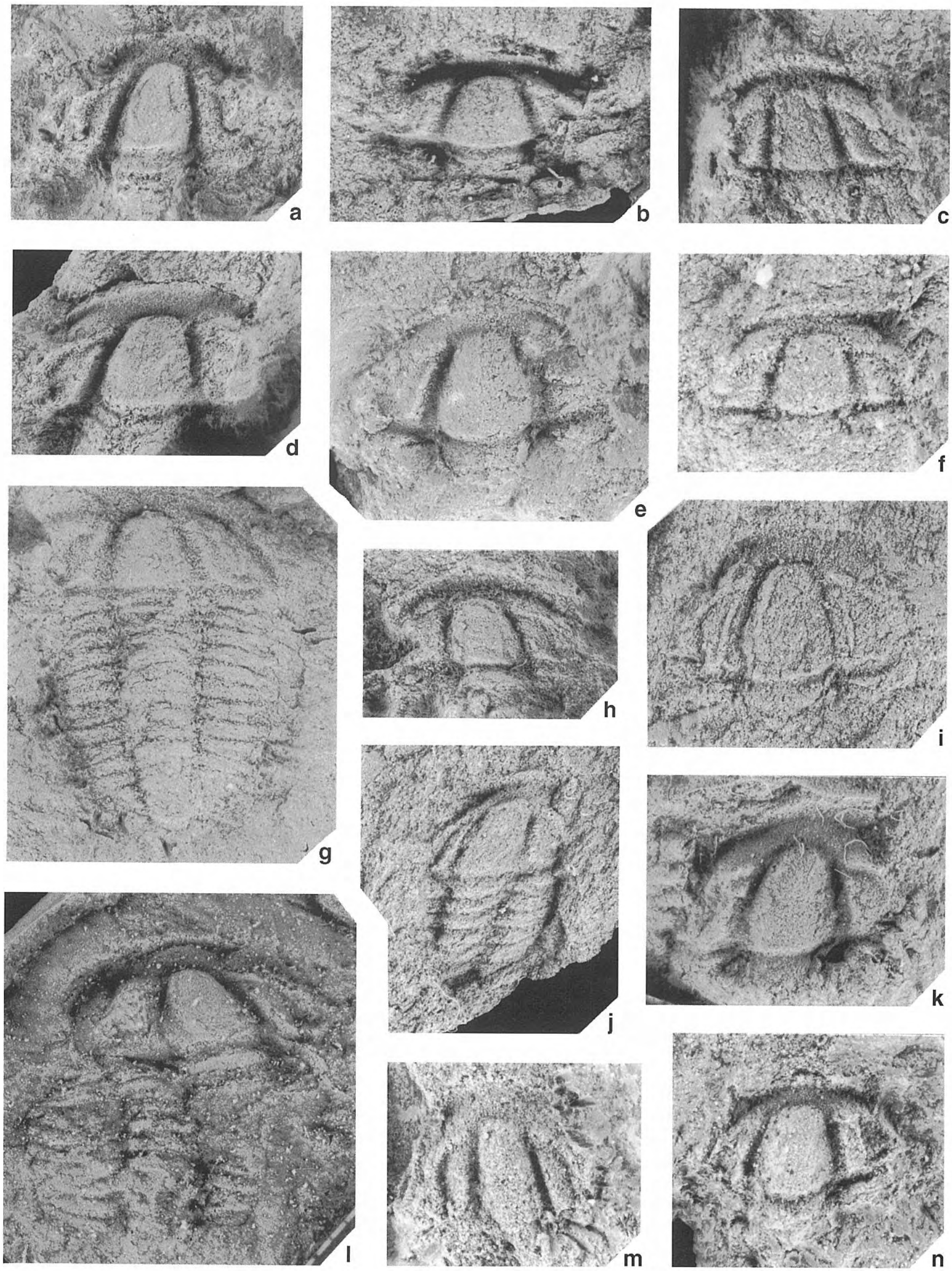

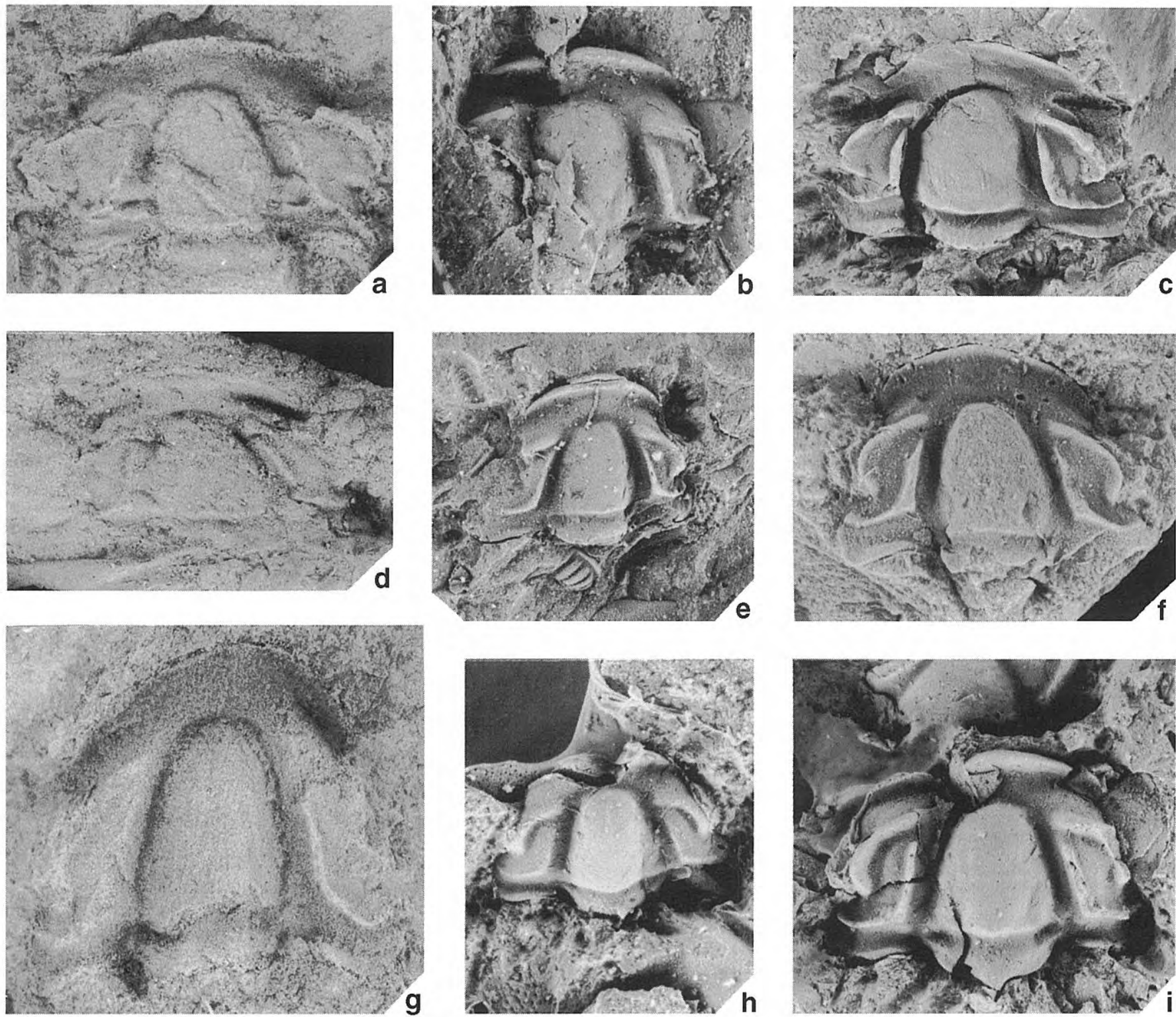

Figura 4. Badulesia tenera (Hartt in Dawson, 1868). a: cranidio, morfotipo B, molde interno (MPZ2001/165), J1/156 (x4). b: cranidio, morfotipo B, molde de látex (MPZ2001/199), Red Bridge Road (x5). c: cranidio, morfotipo A, molde interno (MPZ2001/194), Exit 113 (x5). d: cranidio, morfotipo B, molde de látex (MPZ2001/171), J1/156 (x4). e: cranidio, morfotipo A, molde interno (MPZ2001/193), se observa un fragmento de un pigidio de Dawsonia dawsoni Hartt in Dawson, 1868, Exit 113 (x5). f: cranidio, morfotipo A, molde interno (MPZ2001/195), Exit 113 (x8). g: cranidio, morfotipo A, molde interno (MPZ2001/190), Vi1/7 (x4). h: cranidio, morfotipo A, molde interno (MPZ2001/197), Red Bridge Road (x8). i: cranidio, morfotipo B, molde interno (MPZ2001/196a), Red Bridge Road (x5).

a: cranidium, morphotype B, internal mould (MPZ2001/165), J1/156 (x4). b: cranidium, morphotype B, latex cast (MPZ2001/199), Red Bridge Road (x5). c: cranidium, morphotype A, internal mould (MPZ2001/194), Exit 113 (x5). $d$ : cranidium, morphotype B, latex cast (MPZ2001/171), J1/156 (x4). e: cranidium, morphotype A, internal mould (MPZ2001/193), and a pygidium of Dawsonia dawsoni Hartt in Dawson, 1868, Exit 113 (x5). f: cranidium, morphotype A, internal mould (MPZ2001/195), Exit 113 (x8). g: cranidium, morphotype A, internal mould (MPZ2001/190), Vil/7 (x4). h: cranidium, morphotype A, internal mould (MPZ2001/197), Red Bridge Road (x8). $i$ : cranidium, morphotype B, internal mould (MPZ2001/196a), Red Bridge Road (x5).

surco anterior estrecho y recto en contra del ancho, profundo y curvado de Badulesia; un borde anterior heterogéneo, ancho y convexo, en contraposición del borde anterior homogéneo, estrecho y en forma de cresta que presenta Badulesia; por último, la ausencia de crestas en las áreas palpebrales y postoculares también separa Braintrella de Badulesia.

Por otro lado, la especie Conocephalites ouangondianus Hartt in Dawson, 1868, fue asignada a este género con dudas por Sdzuy (1968), sin embargo, la 
ausencia de una fuerte cresta interior hace que esta especie no se considere como perteneciente al género Badulesia, de acuerdo con la diagnosis enmendada que se propone en este trabajo. En este mismo sentido, los ejemplares figurados como Badulesia sp. en los trabajos de Gámez et al. (1991) y Gozalo et al. (1993), tampoco pertenecerían al género Badulesia, debido a la ausencia de las crestas interiores.

Distribución estratigráfica y geográfica: El género Badulesia tiene una edad Cámbrico Medio temprano, concretamente Caesaraugustiense inferior y parte basal del Caesaraugustiense medio en la Subprovincia Mediterránea, y se encuentra en la Zona de Eccaparadoxides eteminicus en la Subprovincia Avalónica. Dentro del género, la especie que aparentemente presenta una mayor dispersión geográfica es Badulesia tenera (ver más adelante).

\section{Badulesia tenera (Hartt in Dawson, 1868)} Figs. 3 y 4

* 1868 Conocephalites tener, Hartt, MS.; Hartt en Dawson, 652-653.

1868 Conocephalites neglectus, Hartt, MS.; Hartt en Dawson, 654.

1884 Ptychoparia tener; Walcott, 41, lám. 5: 6-6b.

1887 Liostracus tener; Matthew, 137, lám. 1: 3a-3c.

1923 Ptychoparia barthouxi; Mansuy, 288, lám. 10: $4 \mathrm{a}-\mathrm{g}$.

1926 Sao cf. hirsuta: Lecointre, 110, lám. 12: 13, 14(?).

1942 Andrarina tenera (Hartt); Schmidt, 370-373, lám. 22: 18-20, lám. 23: 1-3, 5-9.

pars 1942 Andrarina quadrata (Hartt); Schmidt, 373-374, lám. 23: 4, 15.

1944 Andrarina tenera; Shimer y Shrock, 605, lám. 259: 12-13, lám. 276: 25.

1961 Pardailhania cf. barthouxi (Mansuy); Dean y Krummenacher, 76-80, lám. 10: 1, 2, 4, 7, 9-13.

1968 Badulesia tenera (Hartt en Dawson 1868); Sdzuy, 112-114, lám. 6: 3-12, lám. 8: 7.

1969 Pardailhania cf. barthouxi (Mansuy); Atan, 4, lám. 4: 1 a-b, 2.

1978 Badulesia tenera (Hartt); Skehan et al., fig. 3 a-c. v 1978 Badulesia tenera (Hartt en Dawson, 1868); Liñán, 188-189, lám. 11: 4-6.

v 1982 Badulesia tenera (Hartt en Dawson, 1868); Palacios, 38, lám. 5: 9.

v 1986 Badulesia tenera (Hartt en Dawson, 1868); Liñán y Gozalo, 61, lám. 23: 7-8.

1986 Badulesia tenera (Hartt in Dawson, 1868); Dean et al., 223-224, figs. 10 e, f, h-l.

1988 Badulesia aff. B. tenera (Hartt in Dawson, 1868); Dean in Martin y Dean, 21, lám. 2: 9, 11.

1988 ?Badulesia tenera (Hartt in Dawson, 1868); Dean in Martin y Dean, 21, lám. 1: 10.

v 1991 Badulesia tenera (Hartt en Dawson 1868); Gámez et al., lám. 1: 4.

1993 Badulesia tenera (Hartt); Levi-Setti, 187, lám. 111.

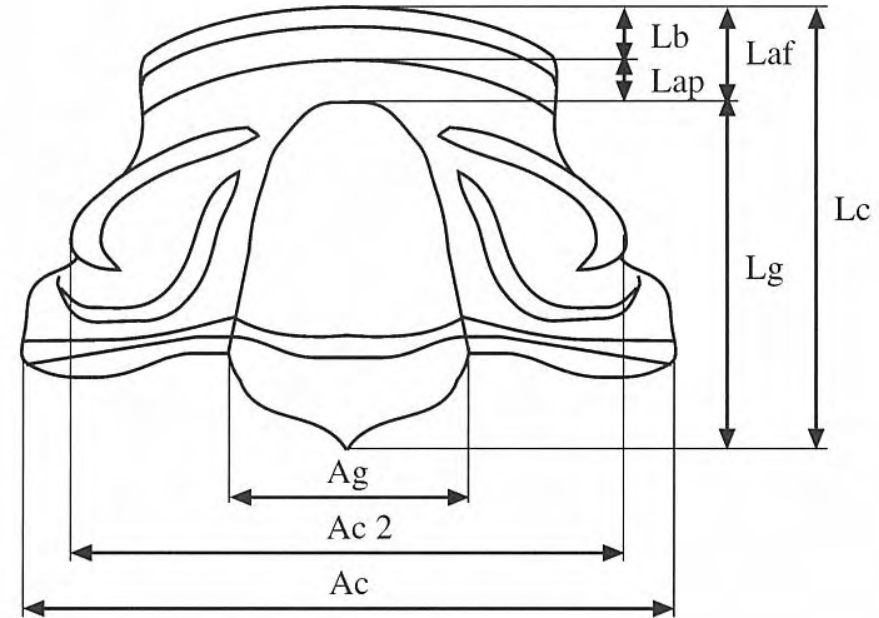

Figura 5. Esquema del cranidio de Badulesia tenera, con indicación de las dimensiones medidas y abreviaturas usadas. Lc, longitud del cranidio; Lg, longitud de la glabela; Lb, longitud del borde anterior del cranidio; Lap, longitud del área preglabelar; Laf, longitud del área frontal; Ac, anchura posterior del cranidio; Ac2, anchura palpebral del cranidio; Ag, anchura de la glabela. Cranidial sketch of Badulesia tenera, with measured dimensions and abbreviations used. Lc, cranidial length; Lg, glabellar length; Lb, cranidium anterior border length; Lap, preglabellar area length; Laf, frontal area length; Ac, posterior cranidial width; Ac2, palpebral cranidial width; Ag, glabellar width.

v 1995 Badulesia tenera (Hartt in Dawson, 1868); Liñán et al., 228, lám. 2: 8-9.

2000 Badulesia tenera (Hartt in Dawson, 1868); Álvaro y Vizcaïno, 281, lám. 1 fig. 12, lám. 2: 12.

Diagnosis enmendada: Especie del género Badulesia con la glabela sin crestas. La fixígena tiene un par de crestas formadas por los lóbulos palpebrales unidos a las aristas oculares y sólo un par de crestas interiores. El área preglabelar puede existir o no.

Species of the genus Badulesia whose glabella lacks ridges. Fixigena has a pair of ridges that are formed by the union of palpebral and eye ridges, and only another inner pair of ridges. The preglabellar field can or cannot appear.

Material y tipo de roca: Unos doscientos cranidios, nueve cefalones y siete ejemplares con el toráx incompleto, en forma de moldes internos y externos, que en muchas ocasiones conservan el delgado exoesqueleto cloritizado; todos ellos procedentes de la sección Jarque 1 (ver Fig. 2). Los especímenes proceden generalmente de lutitas violáceas, ocasionalmente verdes, y también de algún nódulo carbonatado, de la parte alta de la Formación Mansilla y de la base de la Formación Murero. 

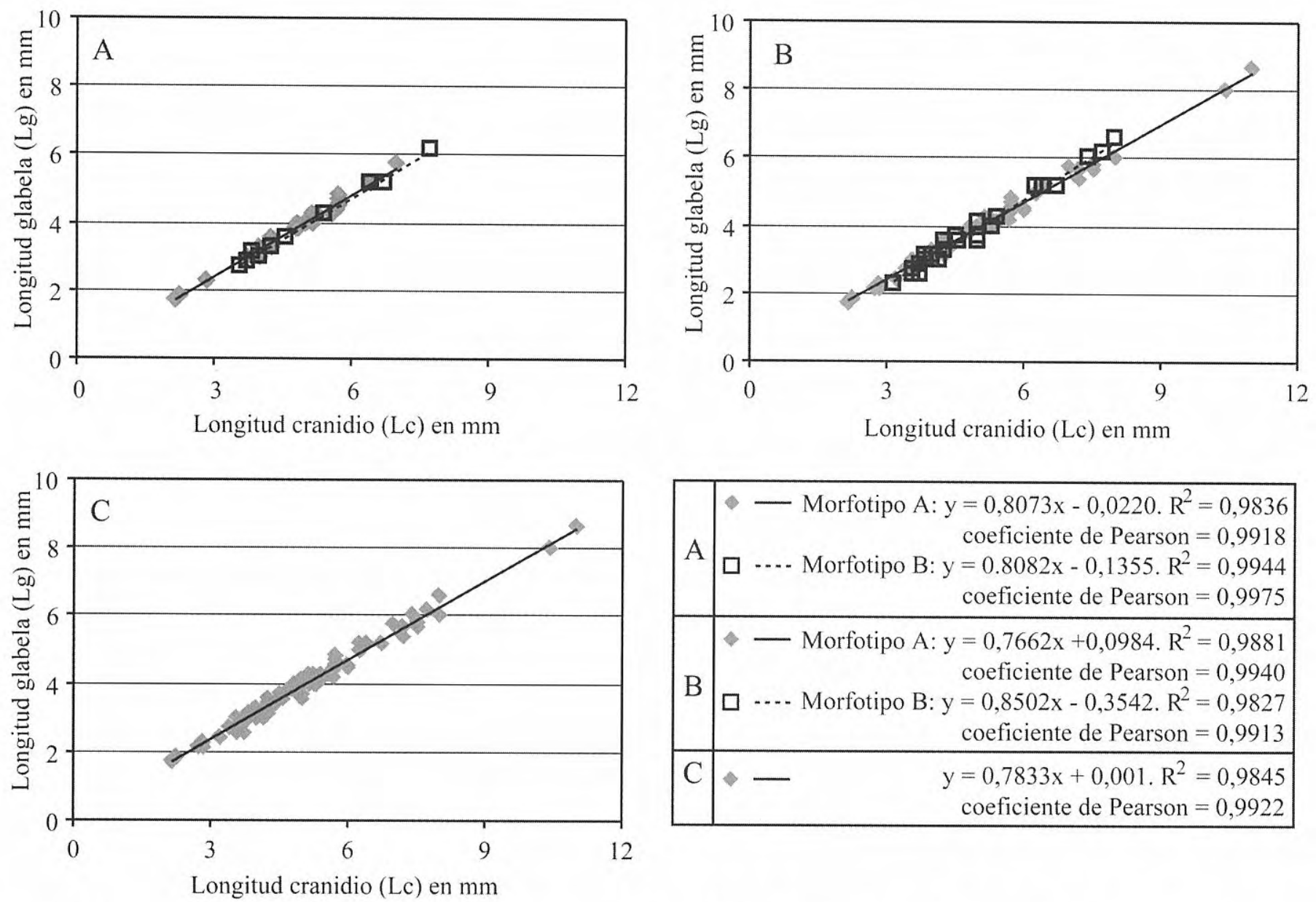

Figura 6. Diagramas de comparación entre Lc y Lg, con indicación de las ecuaciones de las rectas de crecimiento y sus coeficientes de correlación de Pearson y de determinación $\left(\mathrm{R}^{2}\right)$. A: ejemplares del nivel J1/156, con indicación de morfotipos. B: todos los ejemplares medidos, con indicación de morfotipos. C: todos los ejemplares medidos.

Bivariate plots of relationships between $L c$ and $L g$, showing the growth lines, their equations, the Pearson correlation coefficient and the determination coefficient $\left(R^{2}\right)$. A: specimens from level J1/156, showing the two morphotypes. B: all specimens mesured, showing the two morphotypes. C: all specimens mesured.

Siete cranidios y un espécimen con cranidio articulado a cinco segmentos torácicos, conservados como moldes internos y externos, y con restos del exoesqueleto limonitizado; todos ellos procedentes del nivel 13.1 de la sección Rambla de Valdemiedes 2 (ver Liñán y Gozalo, 1986: figura 10). La roca es una marga dolomítica de colores verdes y ocres claros del techo de la Formación Mansilla.

Cuatro cranidios conservados como moldes internos y externos, con restos del exoesqueleto cloritizado; procedentes de los niveles 6 y 7 de la sección Villafeliche 1 (ver Gozalo et al, 1993: figura 3), registrados en lutitas verdes y dolomías ocres de los niveles basales de la Formación Murero.

Seis cranidios conservados como moldes internos y externos, con pátinas limonitizadas; el material prodede de la localidad Exit 113 en la Route 1 de New Brunswick, en las cercanías de la ciudad de Saint John (Landing y Westrop, 1998: 68-70, fig. 28; Westrop y Landing, 2000: fig. 4). Los materiales son lutitas negras y grises oscuras, con algún nódulo carbonatado, pertenecientes a la parte inferior del Miembro Fossil Brook de la Formación Chamberlain's Brook.
Ocho cranidios conservados como moldes internos y externos, algunos de ellos conservan parte del caparazón original, que fueron recolectados en la sección Red Bridge Road en Terranova (ver Landing y Westrop, 1998: 18-22, fig. 5). Los materiales son lutitas negras con nódulos carbonatados, pertenecientes al Miembro Fossil Brook de la Formación Chamberlain's Brook.

\section{Descripción}

Cefalón semicircular, margen anterior y lateral arqueado, margen posterior recto. Borde en forma de cresta submarginal que recorre todo el borde anterior y lateral del cefalón, terminando en el ángulo genal.

Cranidio trapezoidal con sutura opistopárica, las ramas anterior y palpebral de la sutura tienen una longitud aproximadamente equivalente y la rama posterior es algo más larga, con el parámetro de sutura convergente y externo al lóbulo palpebral. El borde es estrecho y elevado en forma de cresta, que se une al surco anterior, que es ancho y profundo, formando una amplia rampa verticalizada. El área preglabelar generalmente no existe; cuando existe está deprimida respecto a la glabela y al borde anterior, siendo 

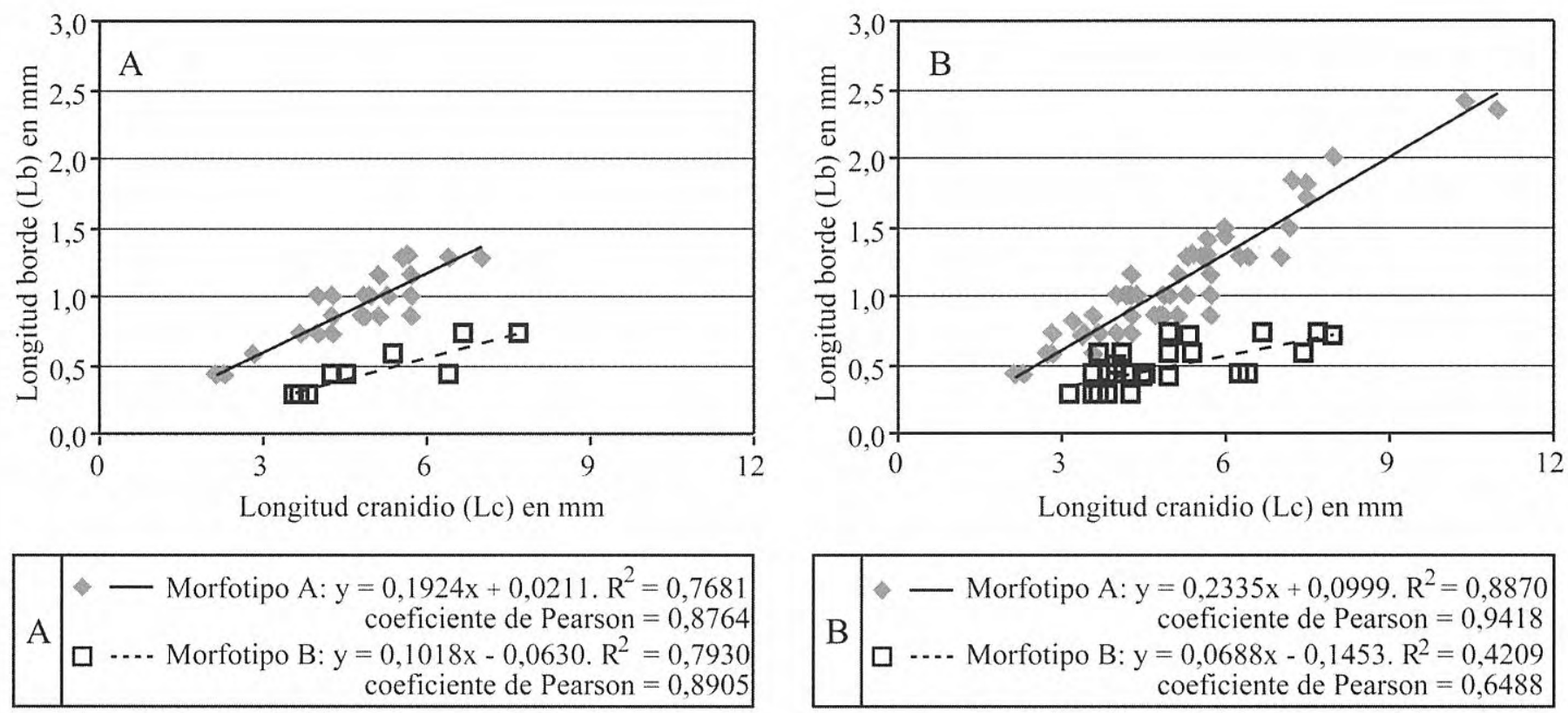

Figura 7. Diagramas de comparación entre Lc y Lb, con indicación de las ecuaciones de las rectas de crecimiento y sus coeficientes de correlación de Pearson y de determinación $\left(\mathrm{R}^{2}\right)$. A: ejemplares del nivel J1/156, con indicación de morfotipos. B: todos los ejemplares medidos, con indicación de morfotipos.

Bivariate plots of relationships between $L c$ and Lb, showing the growth lines, their equations, the Pearson correlation coefficient and the determination coefficient $\left(R^{2}\right)$. A: specimens from level J1/156, showing the two morphotypes. B: all specimens mesured, showing the two morphotypes.

ligeramente convexa y delimitada por un surco preglabelar muy débil, lo que permite establecer dos morfotipos de cranidios dentro de la especie (ver más adelante). Área preocular estrecha que casi desaparece en los ejemplares sin área preglabelar. La glabela es troncocónica, con su parte anterior suavemente redondeada; el surco axial está bien marcado; generalmente es lisa y no presenta crestas; en algunos ejemplares se observan tres surcos poco marcados, no transglabelares y dirigidos hacia atrás. El anillo occipital está bien diferenciado y presenta, en su parte posterior, una expansión triangular a modo de espina occipital; surco occipital neto y recto o ligeramente convexo hacia la parte anterior. Fixígena con dos pares de crestas; el par ocular, externo, formado por la unión de la cresta ocular y del lóbulo palpebral que se inicia a la altura de la parte anterior de la glabela. El segundo par, interno, se localiza en las áreas palpebrales y postoculares. La cresta interior se inicia justo por debajo de la cresta ocular, sin unirse a ella, y transcurre paralela a la glabela y al borde posterior. El surco posterior es amplio y marcado.

Morfotipo A (Fig. 3.a-3.d, 3.f, 3.h, 3.k-3.1, 4.c, 4.e-4.h): el cranidio no presenta área preglabelar, desarrollándose un amplio surco anterior que conecta directamente con el surco preglabelar, el borde anterior se eleva hacia delante formando una rampa, la inclinación de la rampa presenta cierta variación, pues en algunos casos la altura del borde es igual a la de la glabela, mientras que en la mayoría de los casos es menor.

Morfotipo B (Fig. 3.e, 3.g, 3.i-3.j, 3.m-3.n, 4.a-4.b, 4.d, 4.i): el cranidio presenta un surco anterior, ligeramente marcado, que delimita el borde anterior del área preglabelar que varía entre plana y ligeramente convexa. El borde se eleva hacia delante en forma de rampa desde el surco anterior. En este caso también se observan pequeñas variaciones en la inclinación del borde; la altura del borde no llega a sobrepasar la altura de la glabela, al igual que en el morfotipo A; siempre el área preglabelar se sitúa en un plano inferior a la glabela y al borde anterior.

Librígena con el ángulo genal redondeado. El borde lateral tiene forma de una cresta submarginal que termina en el ángulo genal. El área lateral es lisa con una anchura aproximadamente doble de la del borde lateral.

Tórax: aunque el material hallado en las Cadenas Ibéricas no permite su estudio detallado debido a su mala conservación, sus características coinciden con las previamente descritas por Sdzuy (1968). El ejemplar más completo de las Cadenas Ibéricas tiene 14 segmentos torácicos, aunque el número total de segmentos debe de ser 17 (ver Sdzuy, 1968: 114). En los segmentos no se observa la presencia de crestas o espinas, pero algunos de los tórax figurados por Schmidt (1942) y Dean et al. (1986) muestran una espina axial en el raquis de los segmentos. El raquis es abombado y con una anchura ligeramente inferior a la de las pleuras. Las pleuras están dirigidas en su parte final hacia la región anterior, con un surco pleural bien marcado y su terminación es redondeada.

\section{Mudas}

En el material existe algún ejemplo de muda, como es el caso de la figura 3.1. En este ejemplar se observa que el cefalón y el primer segmento torácico permanecen unidos, mientras que los ocho segmentos torácicos posteriores están desplazados y articulados entre sí. Esta disposición parece indicar que la muda se produciría en 

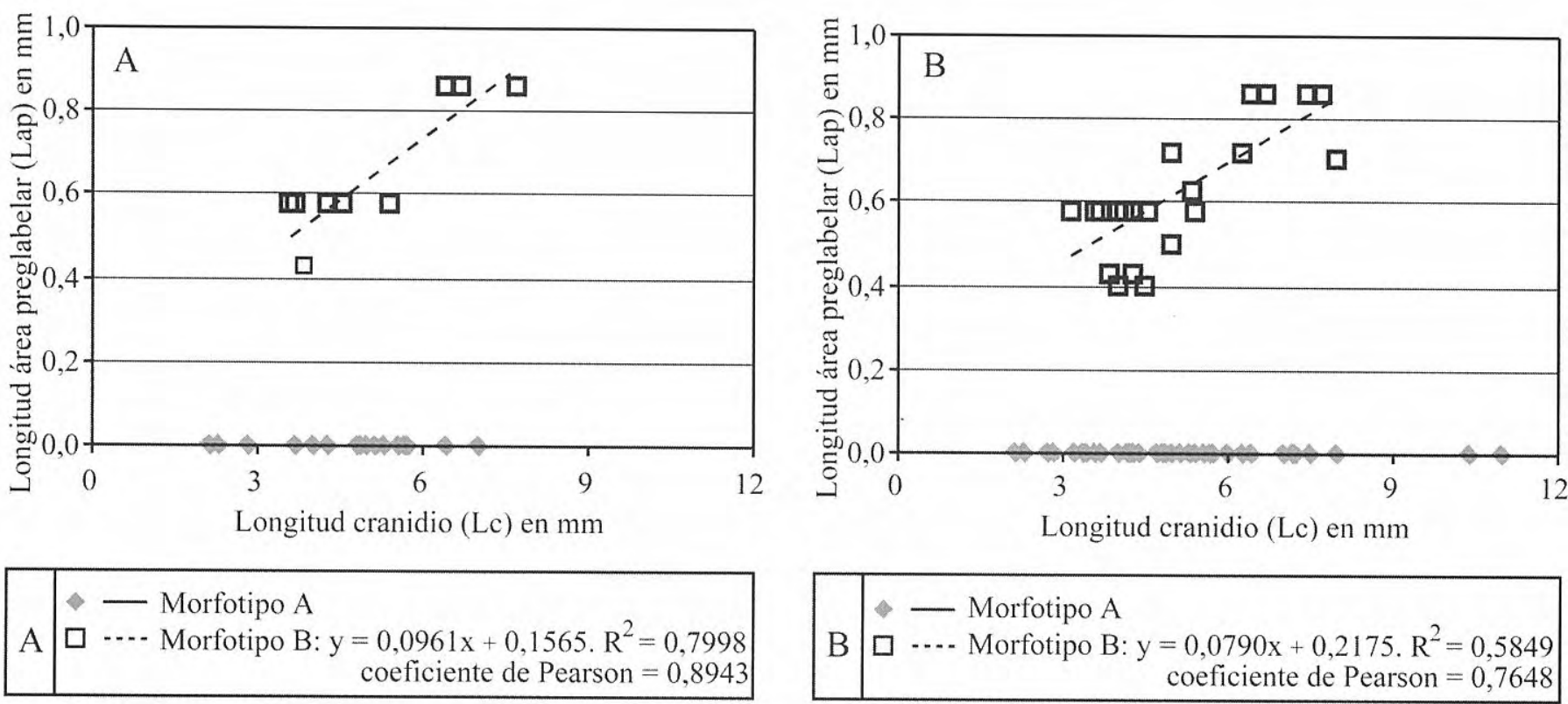

Figura 8. Diagramas de comparación entre Lc y Lap, con indicación de las ecuaciones de las rectas de crecimiento y sus coeficientes de correlación de Pearson y de determinación $\left(\mathrm{R}^{2}\right)$. A: ejemplares del nivel J1/156, con indicación de morfotipos. B: todos los ejemplares medidos, con indicación de morfotipos.

Bivariate plots of relationships between Lc and Lap, showing the growth lines, their equations, the Pearson correlation coefficient and the determination coefficient $\left(R^{2}\right)$. A: specimens from level J1/156, showing the two morphotypes. B: all specimens mesured, showing the two morphotypes.

dos fases; una primera, probablemente, con la separación de los segmentos torácicos posteriores y el pigidio, que no se ha encontrado en ningún ejemplar; y una segunda fase, donde se termina de liberar el exuvio, en la que puede desarticularse el exoesqueleto entre el primer y segundo segmento torácico.

\section{Variabilidad}

Además de los dos morfotipos de cranidios ya comentados, se ha figurado y descrito la presencia de diversas variaciones en las crestas de la fixígena de algunos cranidios; así, Sdzuy (1968) hace referencia a ejemplares con una tercera cresta incipiente en la fixígena y la presencia, en el ángulo de la cresta interior, de una pequeña prolongación de la misma hacia el surco axial de la glabela; Dean et al. (1986) figuran un ejemplar (figs. $10 . \mathrm{i}, 10 . \mathrm{j}$ ) que muestra claramente una tercera cresta en la parte derecha de la fixígena, subparalela a la cresta ocular, mientras que en la parte izquierda se observa una cresta incipiente junto a la cresta interior. Estas variaciones son utilizadas por Álvaro y Vizcaïno (2000) para distinguir varios tipos dentro de esta especie.

En el material estudiado también se han encontrado ejemplares con diferentes morfologías, tales como la presencia de una corta espina genal en las librígenas; la variación de la morfología del ángulo de la cresta interior, siendo en unos ejemplares más redondeado y en otros más anguloso; y el relieve más o menos marcado de las crestas. También la forma de la glabela presenta variaciones, observándose ejemplares que tienen la parte anterior de la glabela poco redondeada, casi recta, mientras que en otros, la glabela presenta su región anterior semicircular e incluso apuntada.

\section{Análisis morfométrico}

En la figura 5 se representa un esquema del cranidio de Badulesia tenera, indicando las dimensiones medidas sobre el mismo. Un aspecto remarcable es que la dimensión Lb en los cranidios del Morfotipo A es coincidente con la dimensión Laf, ya que al no presentar área preglabelar se ha considerado que el borde comienza en el surco preglabelar.

En el Apéndice se ofrecen los datos, tanto de las dimensiones como de los índices, del material utilizado en el análisis morfométrico, que incluye el material estudiado procedente de las secciones Jarque 1 (MPZ2001/131MPZ2001/189 y MPZ2001/198), Villafeliche 1 (MPZ MPZ2001/190) y Rambla de Valdemiedes 2 (MPZ2001/191-MPZ2001/192), y de las localidades Exit 113 (Newbrunswick; MPZ2001/193-MPZ2001/195) y Red Bridge Road (Terranova; MPZ2001/196-MPZ2001/197 y MPZ2001/199); por último, se recogen las medidas de ejemplares previamente publicados de la Cordillera Cantábrica (Sdzuy, 1968), Turquía (Dean et al., 1986) y Terranova (Levi-Setti, 1993). Hay que indicar que el número de ejemplares medidos es menor que el número total de ejemplares estudiados, ya que sólo se han seleccionado aquellos ejemplares en los que, por su conservación, era posible medir todas las dimensiones de un modo fiable.

Para el estudio morfométrico de la especie Badulesia tenera se ha utilizado el análisis bivariante. En los últimos años, este tipo de análisis se ha aplicado a los trilobites cámbricos con dos propósitos principales: el primero para predecir los efectos de la deformación tectónica sobre la morfología de los trilobites (Hughes y Jell, 1992), y el segundo para examinar la relación entre dimensiones durante el crecimiento y obtener las correspondientes líneas de crecimiento (Labandeira y Hughes, 1994; Hughes, 1994). 

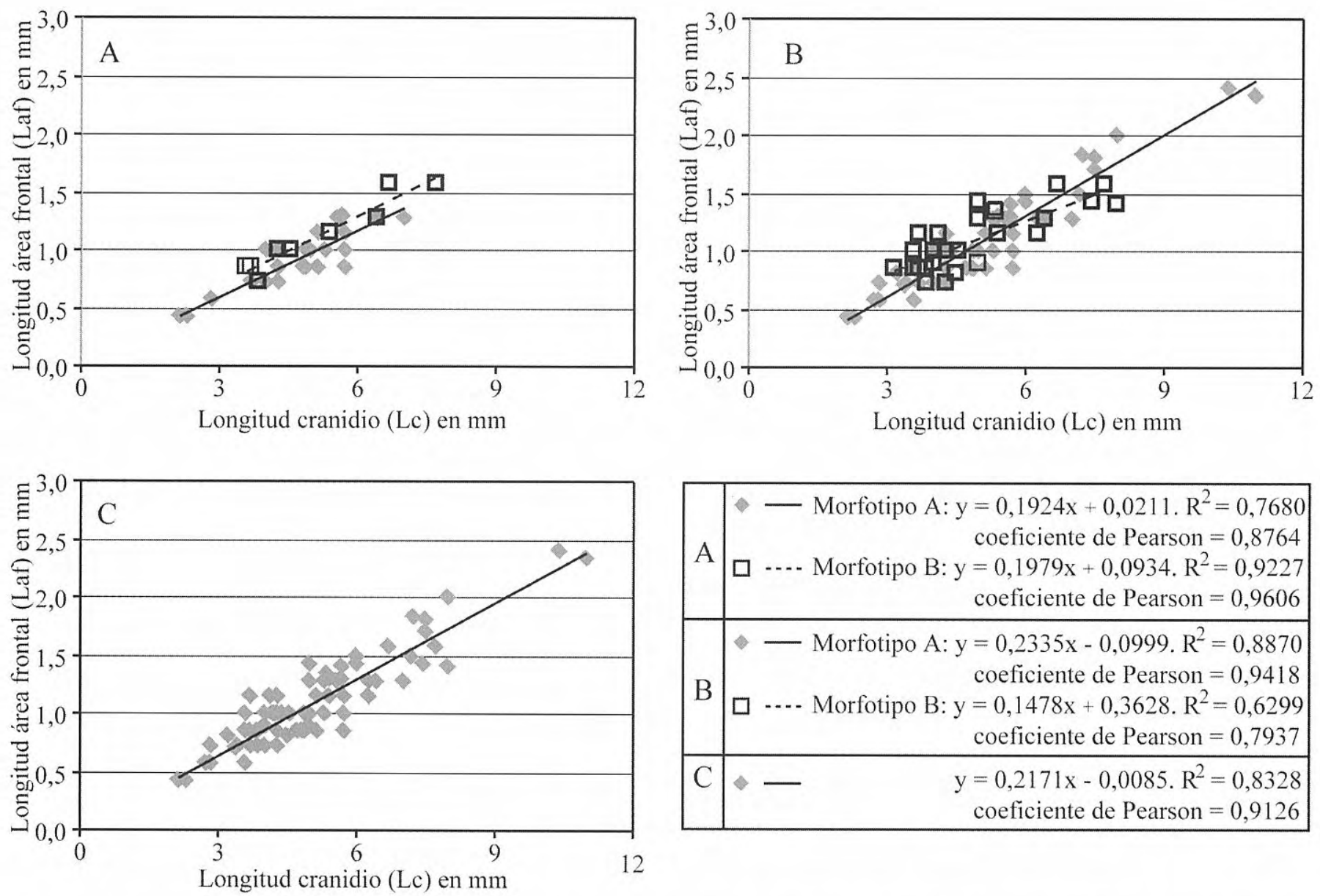

\begin{tabular}{|c|c|c|}
\hline A & $\square$ & $\begin{array}{r}\text { Morfotipo A: } y=0,1924 x+0,0211 . R^{2}=0,7680 \\
\text { coeficiente de Pearson }=0,8764 \\
\text { Morfotipo B: } y=0,1979 x+0,0934 . R^{2}=0,9227 \\
\text { coeficiente de Pearson }=0,9606\end{array}$ \\
\hline B & $\diamond$ & $\begin{array}{r}\text { Morfotipo A: } y=0,2335 x-0,0999 . R^{2}=0,8870 \\
\text { coeficiente de Pearson }=0,9418 \\
\text { Morfotipo B: } y=0,1478 x+0,3628 . \mathrm{R}^{2}=0,6299 \\
\text { coeficiente de Pearson }=0,7937\end{array}$ \\
\hline $\mathrm{C}$ & $\diamond$ & $\begin{array}{r}y=0,2171 x-0,0085 \cdot R^{2}=0,8 \\
\text { coeficiente de Pearson }=0,9\end{array}$ \\
\hline
\end{tabular}

Figura 9. Diagramas de comparación entre Lc y Laf, con indicación de las ecuaciones de las rectas de crecimiento y sus coeficientes de correlación de Pearson y de determinación $\left(\mathrm{R}^{2}\right)$. A: ejemplares del nivel J1/156, con indicación de morfotipos. B: todos los ejemplares medidos, con indicación de morfotipos. C: todos los ejemplares medidos.

Bivariate plots of relationships between Lc and Laf, showing the growth lines, their equations, the Pearson correlation coefficient and the determination coefficient $\left(R^{2}\right)$. A: specimens from level J1/156, showing the two morphotypes. B: all specimens mesured, showing the two morphotypes. $C$ : all specimens mesured.

La utilización de este análisis en materiales deformados tectónicamente ha permitido reconocer que la dispersión observada en estas muestras es debida, en parte, a la propia deformación. Hughes y Jell (1992) demostraron que los coeficientes de correlación de Pearson entre dimensiones medidas en la misma dirección presentan valores superiores que los determinados para dimensiones medidas en direcciones perpendiculares. En la tabla 1 del trabajo citado, estos autores ofrecen los datos del coeficiente de correlación de Pearson para el material que estudian; en ella se destaca que mientras que las dimensiones medidas en la misma dirección presentan coeficientes superiores a 0,8 , superando en muchas ocasiones 0,9 , cuando se trata de dimensiones medidas en direcciones perpendiculares, estos coeficientes descienden a cifras que varían entre 0,64 y 0,8 .

Desde el punto de vista de la deformación, tampoco hay que olvidar el efecto de la compactación. Por ejemplo, Webster y Hughes (1999) analizan este efecto para algunas especies de olenéllidos, y una de las conclusiones que ofrecen es que el simple hecho de que el material se encuentre aplastado hace que algunos puntos de referencia (landmark) sufran cambios significativos, sobre todo con variaciones laterales y verticales importantes, lo que también conlleva una mayor dispersión de estos puntos cuando se reflejan en un diagrama.

El otro aspecto analizado a través de la comparación entre dos dimensiones es establecer las correspondientes líneas de crecimiento para las dimensiones utilizadas; en este caso, cuando los coeficientes de correlación de Pearson son elevados, son indicativos de que el crecimiento es el principal control de la variación entre esas dos dimensiones (Labandeira y Hughes, 1994). Un aspecto a tener en cuenta es que la mayoría de las poblaciones estudiadas presentan un amplio rango de exuvios de distintos estadios (instars) de holaspis, pero no de otros estadios embrionarios, por lo que se suelen obtener rectas de crecimiento que indicarían crecimientos isómetricos para los holaspis. Labandeira y Hughes (op. cit.) proponen que si la recta de crecimiento corta aproximadamente en el origen, se trataría realmente de un crecimiento isométrico, en caso contrario, muy probablemente se trataría de un crecimiento alométrico, aunque para confirmar esta cuestión sería necesario disponer de especímenes de los estadios protaspis y meraspis. También estos autores indican que una dispersión importante de los 

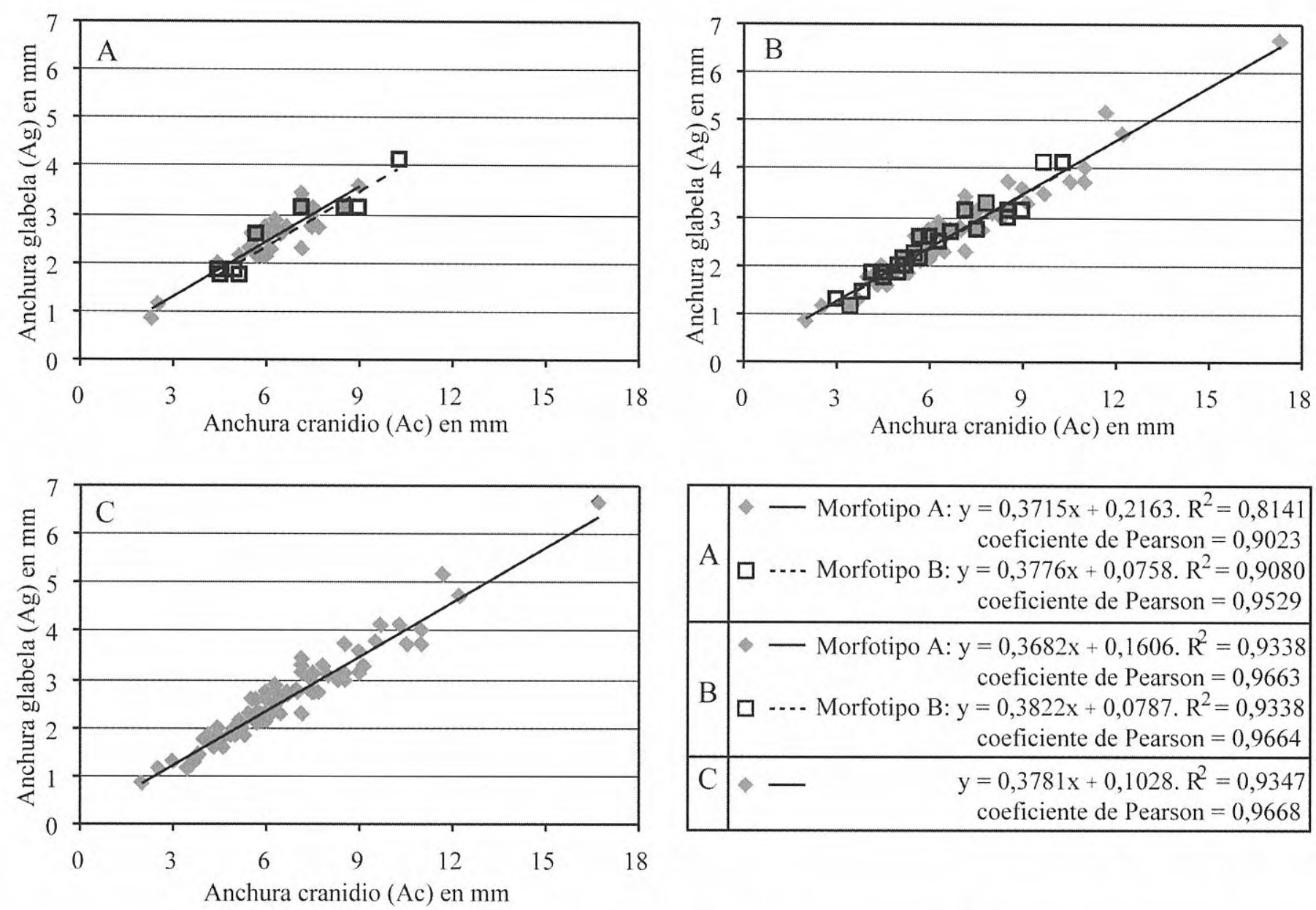

Figura 10. Diagramas de comparación entre Ac y Ag, con indicación de las ecuaciones de las rectas de crecimiento y sus coeficientes de correlación de Pearson y de determinación $\left(\mathrm{R}^{2}\right)$. A: ejemplares del nivel J1/156, con indicación de morfotipos. B: todos los ejemplares medidos, con indicación de morfotipos. C: todos los ejemplares medidos.

Bivariate plots of relationships between Ac and Ag; showing the growth lines, their equations, the Pearson correlation coefficient and the determination coefficient $\left(R^{2}\right)$. A: specimens from level J1/156, showing the two morphotypes. B: all specimens mesured, showing the two morphotypes. $C$ : all specimens mesured.

puntos respecto a la línea de crecimiento, esto es, un coeficiente de correlación bajo, indicaría que el control del crecimiento para estas dimeñsiones era más flexible que para otras. Otro aspecto reseñable es que la continuidad morfológica, ya sea de una población o cuando se comparan varias poblaciones, sería una evidencia positiva de la integridad de una especie, como expresan Labandeira y Hughes (1994: 501): "While it is not possible to discriminate distinct species from this morphometric analysis, it is clear that there is substancial morphological variation within the sample".

Esta técnica ha sido utilizada en diferentes trabajos para revisiones sistemáticas, lo que ha llevado a poner en sinonimia especies, e incluso géneros, que previamente se habían considerado diferentes, pero que el análisis morfométrico ha constatado que las variaciones se debían exclusivamente a las diferencias de tamaños y a la deformación tectónica (Hughes y Jell, 1992; Hughes, 1994; Rushton y Hughes, 1996).

En las figuras 6 a 13 se presentan los diagramas de comparación entre dos dimensiones para los dos morfotipos de la población del nivel J1/156 (A), para los dos morfotipos de todos los ejemplares medidos (B) y para el conjunto de los ejemplares medidos sin diferenciar morfotipos (C); también se incluyen las ecuaciones de las rectas de regresión obtenidas, que corresponderían a rectas de crecimiento de acuerdo con las propuestas de Labandeira y Hughes (1994) y Hughes (1994), así como los coeficientes de correlación de Pearson y el de determinación $\left(\mathrm{R}^{2}\right)$, para cada una de las ecuaciones. Los coeficientes obtenidos son coherentes con la consideración de la variabilidad observada como intraespecífica.

En la figura 6 se comparan la dimensión Lc con la Lg; todas las rectas obtenidas muestran un coeficiente de correlación de Pearson superior a 0,99 y el de determinación superior a 0,98 , lo que indica que la variación entre ambas dimensiones estaría controlada por el crecimiento dentro de una misma especie.

En la figura 7 se compara Lc con Lb; en este caso, al depender Lb de la existencia o no de área preglabelar, se observa una separación clara entre los puntos que representan a ambos morfotipos. Las rectas obtenidas también presentan pendientes muy dispares y los 

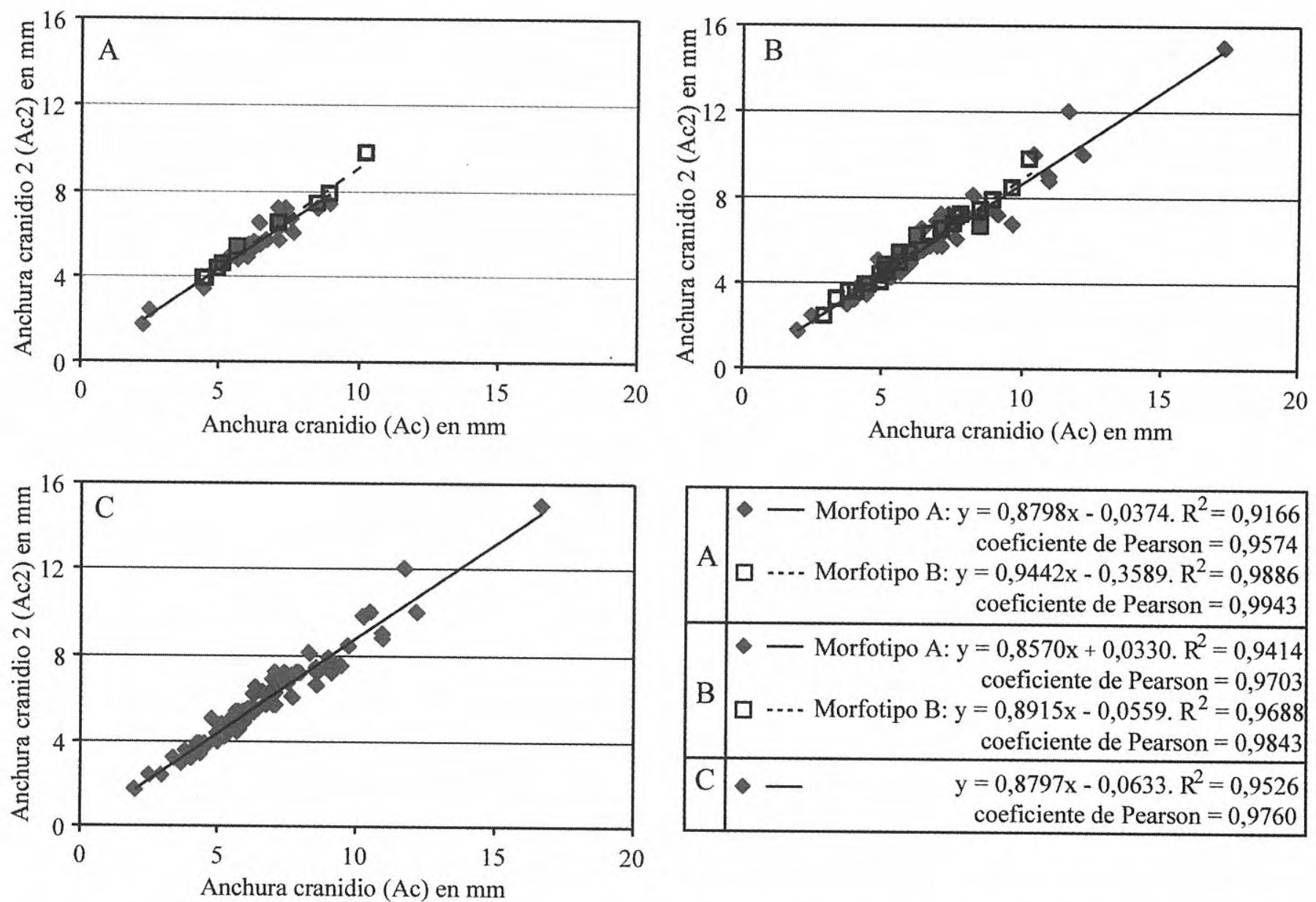

Figura 11. Diagramas de comparación entre Ac y Ac2, con indicación de las ecuaciones de las rectas de crecimiento y sus coeficientes de correlación de Pearson y de determinación $\left(\mathrm{R}^{2}\right)$. A: ejemplares del nivel J1/156, con indicación de morfotipos. B: todos los ejemplares medidos, con indicación de morfotipos. C: todos los ejemplares medidos.

Bivariate plots of relationships between Ac and Ac2, showing the growth lines, their equations, the Pearson correlation coefficient and the determination coefficient $\left(R^{2}\right)$. A: specimens from level J1/156, showing the two morphotypes. B: all specimens mesured, showing the two morphotypes. C: all specimens mesured.

coeficientes de Pearson y $\mathrm{R}^{2}$ son relativamente bajos, lo que permite suponer que el control del crecimiento, en este caso, no es muy estricto y, por lo tanto, posibilitaría una amplia variación. En la figura 8 se compara Lc con Lap; en este caso, la ausencia del área preglabelar en el Morfotipo A hace que todos sus puntos se situen sobre el eje de abscisas, mientras que los coeficientes de Pearson y $\mathrm{R}^{2}$ para el Morfotipo B, son también bajos, lo que, como en el caso anterior, podría indicar la existencia de una importante variabilidad. Como se constata en estas dos figuras, existe una clara separación morfométrica entre los dos morfotipos establecidos por criterios morfológicos.

En la figura 9 se compara Lc con Laf; en este caso se observa que, aunque los coeficientes de Pearson y $\mathrm{R}^{2}$ son más elevados que en los dos casos anteriores, siguen siendo relativamente bajos cuando se analizan para los morfotipos por separado, y las rectas obtenidas presentan diferencias claras en las pendientes; todo ello parece indicar que aunque el control del crecimiento en la variación de estas dos dimensiones es importante en el conjunto de la especie, este control es menor dentro de cada uno de los morfotipos, lo que implicaría la posibilidad de una variabilidad importante en la morfología de la región anterior del cranidio para cada morfotipo.

En las figuras 10 y 11 se comparan Ac con Ag y con Ac2, respectivamente. En ambos casos se observan unas pendientes similares para las rectas de crecimiento y unos coeficientes de Pearson y $\mathrm{R}^{2}$ elevados, superiores a 0,95 y 0,9 , respectivamente, excepto en un caso, por lo que la variación entre estas dimensiones muestra un claro control del crecimiento, que es congruente con la consideración de una única especie.

En las figuras 12 y 13 se comparan dimensiones que se han medido en direcciones perpendiculares, $\mathrm{Lg}$ con $\mathrm{Ag}$ y $\mathrm{Lc}$ con Ac. En ambos casos se observa que sus coeficientes de Pearson y $\mathrm{R}^{2}$, en general, son cercanos o superiores a $0,85 \mathrm{y}$ 0,8 , respectivamente, lo que, teniendo en cuenta que se trata de un material deformado, se puede interpretar como debido a que el control del crecimiento sobre las variaciones en estas dimensiones es importante y seguramente similar al existente para otras dimensiones ya comentadas previamente. 

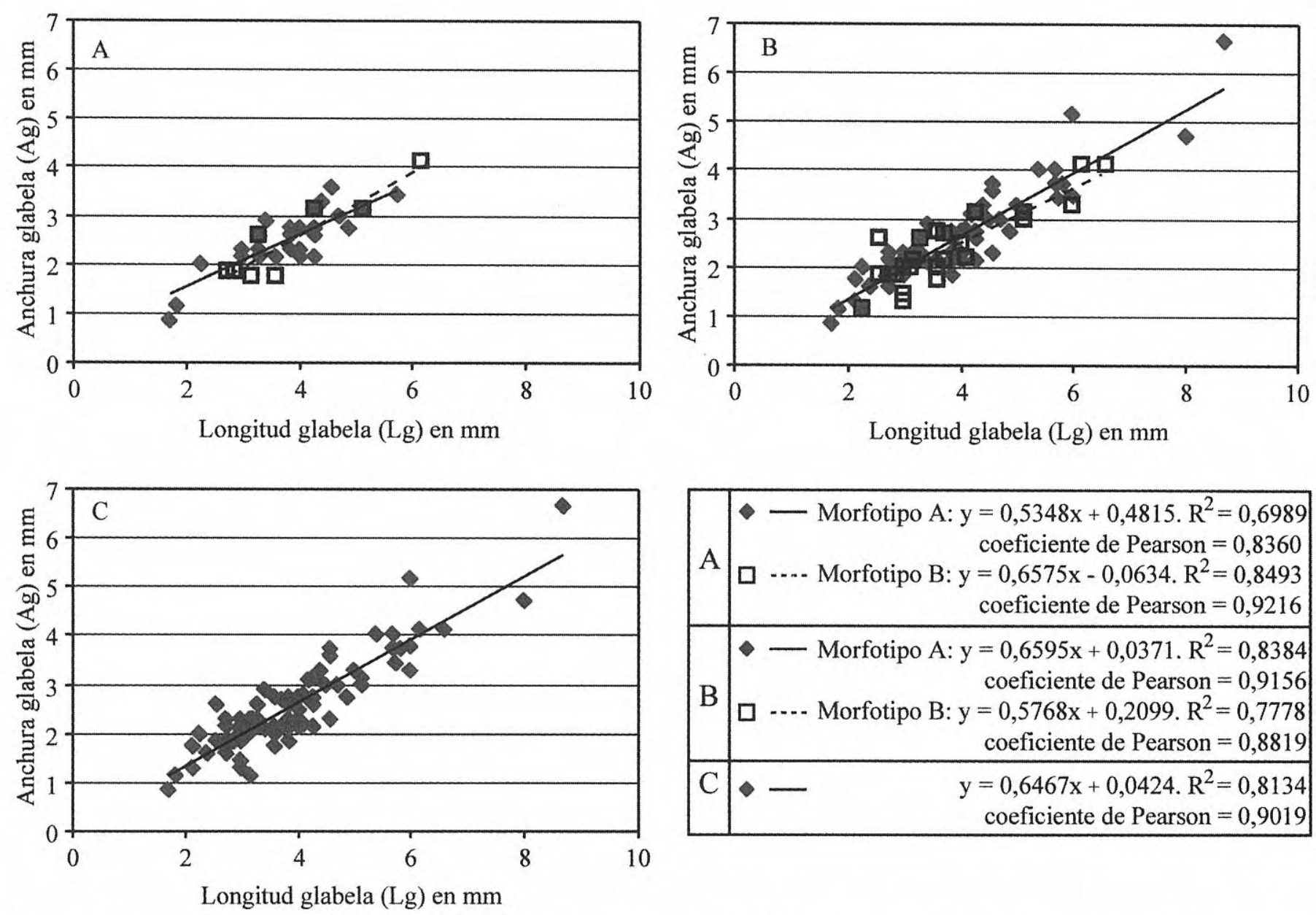

Figura 12. Diagramas de comparación entre $\mathrm{Lg}$ y Ag, con indicación de las ecuaciones de las rectas de crecimiento y sus coeficientes de correlación de Pearson y de determinación $\left(\mathrm{R}^{2}\right)$. A: ejemplares del nivel J1/156, con indicación de morfotipos. B: todos los ejemplares medidos, con indicación de morfotipos. C: todos los ejemplares medidos.

Bivariate plots of relationships between $\mathrm{Lg}$ and $\mathrm{Ag}$, showing the growth lines, their equations, the Pearson correlation coefficient and the determination coefficient $\left(R^{2}\right)$. A: specimens from level J1/156, showing the two morphotypes. B: all specimens mesured, showing the two morphotypes. $C$ : all specimens mesured.

En resumen, se puede decir que todas las dimensiones comparadas, excepto las que involucran las longitudes de la región anterior del cranidio, presentan en general unos buenos coeficientes de correlación de Pearson y de determinación $\left(\mathrm{R}^{2}\right)$ para las rectas de crecimiento calculadas, por lo que la variación de las mismas debe de estar controlada fundamentalmente por el crecimiento dentro de una única especie. También se ha constatado que cuando se comparan dimensiones medidas en direcciones perpendiculares, sus coeficientes de correlación son más bajos que cuando las dimensiones se miden en la misma dirección, tal y como ya planteaban Hughes y Jell (1992). Por contra, cuando la comparación ha sido entre la longitud del cranidio y las longitudes de la región anterior del mismo, se ha constatado la existencia de una variabilidad mucho mayor, observándose una separación clara entre los especímenes asignados a cada uno de los dos morfotipos; en estos casos, tanto los coeficientes de Pearson como $\mathrm{R}^{2}$ son bajos, lo que parece indicar que la independencia de estas dimensiones respecto al crecimiento es mucho mayor que para otras dimensiones y permitiría una variación intraespecífica mucho más amplia.
Por último, en la figura 14 se ha comparado una dimensión con un índice, o bien, dos índices de los dos morfotipos de todo el material medido. En las figuras 14A, 14C y 14D aparecen los puntos de los dos morfotipos claramente mezclados, por lo que se colige que ambos morfotipos forman un solo conjunto y por lo tanto deben ser consideradas como variaciones intraespecíficas. Sin embargo, en la figura $14 \mathrm{~B}$, donde se compara la dimensión Lc con el índice $\mathrm{Lb} / \mathrm{Lc}$, que incluye uno de los elementos utilizados para separar los dos morfotipos, se observa que mientras los ejemplares del morfotipo A presentan valores del índice siempre mayores de 0,15 , los del morfotipo B siempre son menores de 0,15 , por lo que también aquí se constata una clara separación morfométrica entre ambos morfotipos.

\section{Discusión}

Los nuevos ejemplares aquí estudiados han permitido por primera vez el análisis de una muestra poblacional de esta especie proveniente de un sólo nivel y su comparación con las de niveles sucesivos; también establecer un amplio rango de variación para el cranidio, dentro de dos morfotipos, que posibilita incluir en esta 

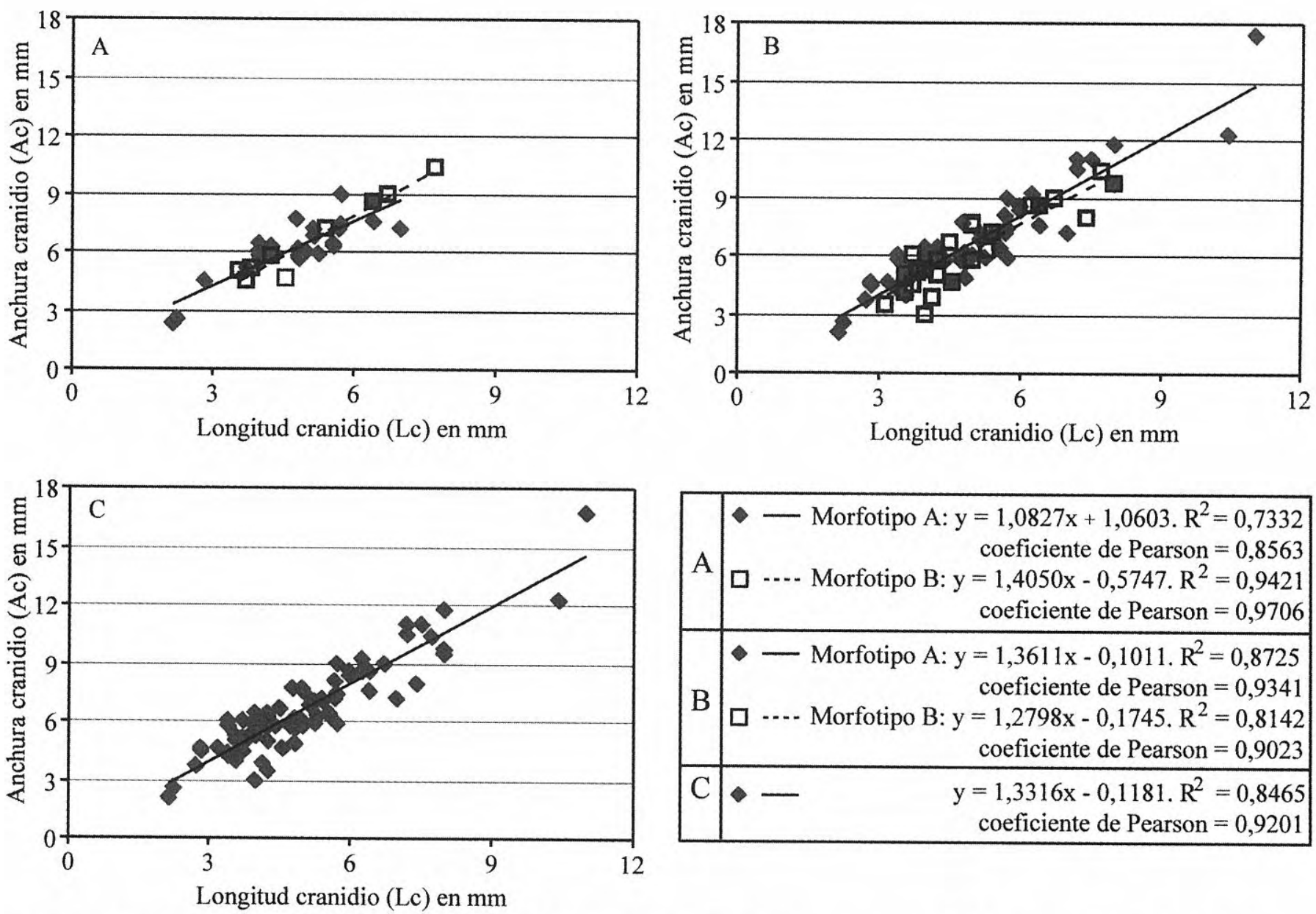

Figura 13. Diagramas de comparación entre Lc y Ac, con indicación de las ecuaciones de las rectas de crecimiento y sus coeficientes de correlación de Pearson y de determinación $\left(\mathrm{R}^{2}\right)$. A: ejemplares del nivel J1/156, con indicación de morfotipos. B: todos los ejemplares medidos, con indicación de morfotipos. C: todos los ejemplares medidos.

Bivariate plots of relationships between Lc and Ac, showing the growth lines, their equations, the Pearson correlation coefficient and the determination coefficient $\left(R^{2}\right)$. A: specimens from level J1/156, showing the two morphotypes. B: all specimens mesured, showing the two morphotypes. $C$ : all specimens mesured.

especie ejemplares que por sus diferencias morfológicas habían sido considerados previamente en nomenclatura abierta, por ej. Badulesia aff. B. tenera sensu Dean in Martin y Dean, 1988. Por otro lado, como ya había expresado Sdzuy (1968), se encuentran numerosos ejemplares que presentan algunas modificaciones en la morfología de las crestas de la fixígena o en la glabela, que parecen una anticipación de los caracteres de futuras especies, por eso se considera que mientras estas modificaciones no sean simétricas en ambos lados de la fixígena es preferible mantenerlas dentro de la variabilidad de una sola especie.

\section{Distribución estratigráfica y geográfica}

Esta especie fue descrita en la subprovincia Avalónica, en la localidad de Saint John (New Brunswick, Canadá) por Hartt in Dawson (1868), en el Miembro Fossil Brook de la Formación Chamberlain's Brook, dentro de la Zona de Eccaparadoxides eteminicus (Landing y Westrop, 1998; Westrop y Landing, 2000). La misma posición estratigráfica ocupa en Terranova (Canadá), de acuerdo con Landing y Westrop (1998). Dentro de esta región biogeográfica también se ha encontrado en Rhode Island (Estados Unidos), concretamente en un nivel dentro de una unidad que Skehan et al. (1978) denominan phyllites on Conanicut Island, la cual datan por la presencia de Badulesia tenera.

Dentro de la Subprovincia Mediterránea, el primer lugar donde se encontró esta especie fue en Marruecos, donde Mansuy (1923) la describió como Ptychoparia barthouxi; posteriormente ha sido citada tanto en el Alto como en el Anti Atlas marroquí, concretamente en la Zona de Badulesia de las formaciones Jbel Wawrmast y Jbel Afraou (Geyer, 1990; Geyer y Landing, 1995; Geyer et al., 1995). Posteriormente se descubrió en Doberlug (Alemania) por Schmidt (1942), habiendo sido revisada esta fauna por Sdzuy (1957). El siguiente hallazgo fue en las montañas de Amanos (Turquía) debido a Dean y Krummenacher (1961), en los niveles basales de la Formación Sosink (Dean et al., 1986; Dean y Monod, 1997). En España, esta especie fue citada por primera vez por Sdzuy (1968) en materiales de la Cordillera Cantábrica que actualmente se consideran pertenecientes a la parte baja de la Formación Oville, de acuerdo con Zamarreño (1972); Sdzuy (1969) amplía su distribución a la Formación Láncara de la Zona Cantábrica, 

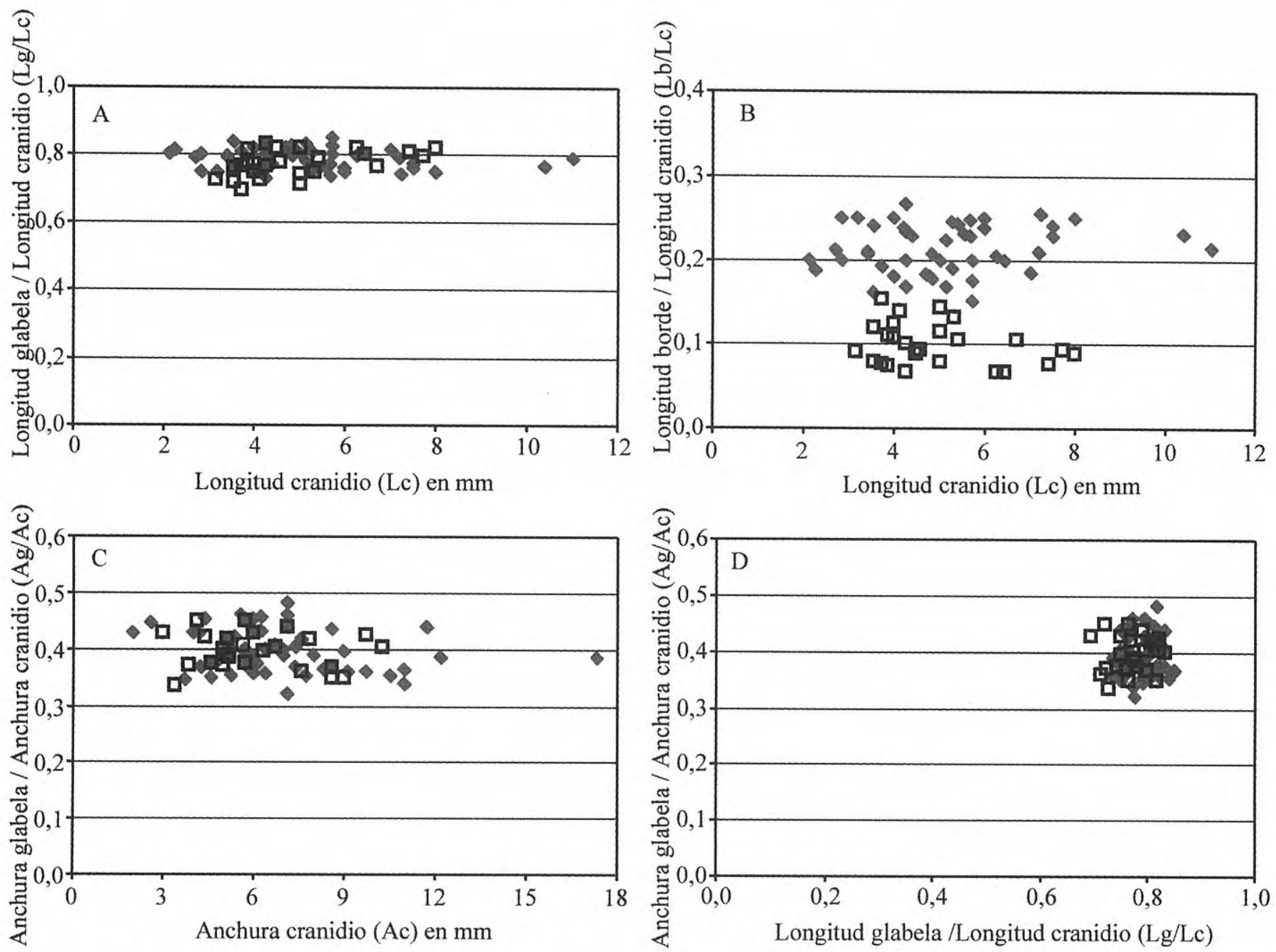

- Morfotipo A $\square$ Morfotipo B

Figura 14. Diagramas de comparación entre un índice con una dimensión u otro índice para todos los ejemplares medidos, con indicación de los dos morfotipos. A: Lc y Lg/Lc. B: Lc y Lb/Lc. C: Ac y Ag/Ac. D: Lg/Lc y Ag/Ac.

Bivariate plots of relationships between one dimension and one ratio or between two ratios. All specimens are represented showing the morphotypes. A: $L c$ versus $L g / L c$. B: $L c$ versus $L b / L c$. C: Ac versus $A g / A c$. $D: L g / L c$ versus $A g / A c$.

a las capas de Riotorto (Serie de Los Cabos) en la Zona Asturoccidental-Leonesa y a la Formación Murero en las Cadenas Ibéricas (ver más adelante); la última área de España en la que se encontró esta especie fue en la Formación Los Villares de la Sierra de Córdoba (Liñán, 1978). Por último, esta especie se ha citado en la Formación Couloma de la Montaña Negra (Francia) por Álvaro y Vizcaïno (2000). En todas estas localidades la presencia de Badulesia tenera se viene considerando como indicativa de la zona del mismo nombre, o bien de los niveles inferiores de la zona de Badulesia granieri cuando se encuentra asociada a esta especie, siempre dentro del Caesaraugustiense Inferior.

La especie Badulesia tenera, en las Cadenas Ibéricas, se ha encontrado en numerosas localidades; la primera referencia fue en la base de la Formación Murero en Badules de acuerdo con Sdzuy (1969). Posteriormente Liñán y Gozalo (1986) la citaron en el nivel 13.1 de la sección Rambla de Valdemiedes 2, en la parte alta de la Formación Mansilla; en una situación estratigráfica similar se encontro en la sección Mesones 3 (Valenzuela et al., 1990). Gámez et al. (1991) citan esta especie en el nivel 9 de la sección Borobia 2, en los niveles basales de la Formación Murero; en la misma posición estratigráfica se encuentra en el nivel 6 de la sección Villafeliche 1 (Gozalo et al., 1993, 1996). Finalmente fue hallada en el nivel 160 de la sección Jarque 1, en el techo de la Formación Mansilla, y en el nivel 175 de la misma sección, en la base de la Formación Murero (Liñán et al., 1996). En este trabajo, además de los niveles citados, se amplía la presencia de la especie al nivel 7 de la sección de Villafeliche 1 en la Formación Murero, y se presenta por primera vez un registro casi continuo desde el nivel 155 hasta el nivel 175 de la sección Jarque 1, que cubre el tránsito entre las formaciones Mansilla y Murero. Todas las referencias estratigráficas a las formaciones han sido homogeneizadas según la propuesta de Liñán et al. (1992).

Los hallazgos precedentes y los nuevos de las Cadenas Ibéricas hacen suponer que la especie $B$. tenera tiene un rango de distribución que incluye la Zona de Badulesia tenera y la parte baja de la Zona de Badulesia granieri 
(Subzona de Badulesia juliverti), ya que por el momento nunca se ha observado la convivencia con Badulesia paschi, taxón que caracteriza la parte superior de la Zona de Badulesia granieri (Subzona de Badulesia paschi). Una correlación general de la Zona de Badulesia tenera puede verse en Sdzuy et al. (1999).

\section{CONCLUSIONES}

El estudio de más de doscientos ejemplares de la especie Badulesia tenera permite comprobar la alta variabilidad morfológica intraespecífica existente en las especies de trilobites del Cámbrico Medio, y por lo tanto la necesidad de realizar estudios poblacionales donde se encuentren representados distintos niveles estratigráficos y localidades.

Los estudios morfológico y morfométrico realizados han permitido precisar los caracteres genéricos y específicos, conduciendo a una más aquilatada caracterización de los mismos.

El muestreo intensivo realizado en la sección Jarque 1 ha permitido mostrar un registro casi continuo de Badulesia tenera desde su aparición, en la base de la zona homónima, hasta su desaparición en la parte inferior de la zona de Badulesia granieri (ambas del Caesaraugustiense inferior).

El estudio morfológico de los ejemplares ha permitido constatar la existencia de dos morfotipos, comprobándose su presencia en las diferentes regiones donde se encuentra esta especie.

Finalmente, el estudio morfométrico pone en evidencia que son posibles las determinaciones taxonómicas con material de moldes tanto internos como externos, así como con material deformado y $\sin$ deformar, y son igual de fiables, siempre y cuando el número de ejemplares permita realizar un estudio poblacional.

\section{AGRADECIMIENTOS}

Los autores agradecen a los Dres. Álvaro (Lille), Arbizu (Oviedo) y Martínez Chacón (Oviedo) la revisión crítica del manuscrito que ha mejorado el texto original. Este trabajo es una contribución al proyecto BTE 2000-1145-C02-01 de la Dirección General de Estudios Superiores y a los proyectos del Departamento de Educación y Cultura de la Diputación General de Aragón 068/98 y 121/99. Los autores agradecen a la Dra. Zarela Herrera la fotografía de los ejemplares figurados.

\section{BIBLIOGRAFÍA}

Álvaro, J.J. et Vizcaïno, D. 2000. Nouvel assemblage de trilobites dans le Cambrien moyen de la nappe de Pardailhan (Montagne Noire, France): implications biostratigraphiques dans la région méditerranéenne. Eclogae geologicae Helvetiae, 93, 277-289.
Angelin, N.P. 1854. Palaontologica Scandinavica. Pars II; Crustacea formationis transitionis. Academie Regiae Scientarium Suecanae, Fasc. 2, I-IX, 21-92, Holmiæ (Stockolm).

Atan, O. 1969. Geology of the Amanos Mountains (Egribucack-Karacaören-Ceylanli-Dasevleri). Madan Tetkik ve Arama Enstituüsü Yayinlarindan, 139, 1-10.

Bengtson, S. and Fletcher, T. P. 1983. The oldest sequence of skeletal fossils in the Lower Cambrian of southeastern Newfoundland. Canadian Journal of Earth Sciences, 20, 525-536.

Dawson, J.W. 1868. Acadian Geology. The geological structure, organic remains and mineral resources of Nova Scotia, New Brunswick, and Prince Edward Island, 2nd ed., Macmillan, London, xxvi+694 pp.

Dean, W.T. and Krummenacher, R. 1961. Cambrian Trilobites from the Amanos mountains. Turkey. Palaentology, 4, 71-81.

Dean, W.T. and Monod, O. 1997. Cambrian development of the Gondwanaland margin in southeastern Turkey. Turkish Association of Petroleum Geologists, Special Publication, 3, 61-74.

Dean, W.T., Monod, O. and Günay, Y. 1986. Lower Palaeozoic Stratigraphy in the Southern and Central Amanos Mountains, south central Turkey. Geological Magazine, 123, 215-226.

Gámez, J.A., Fernández-Nieto, C., Gozalo, R., Liñán, E., Mandado, J. y Palacios, T. 1991. Bioestratigrafía y evolución ambiental del Cámbrico de Borobia (Provincia de Soria, Cadena Ibérica Oriental). Cuadernos do Laboratorio Xeolóxico de Laxe, 16, 251-271.

Geyer, G. 1990. Die marokkanischen Ellipsocephalidae (Trilobita: Redlichiida). Beringeria, 3, 1-363.

Geyer, G. and Landing, E. 1995. The Cambrian of the Moroccan Atlas regions. Beringeria, Special Issue 2, 7 46.

Geyer, G. and Landing, E. 2001. Middle Cambrian of Avalonian Massachusetts: stratigraphy and correlation of the Braintree trilobites. Journal of Paleontology, $\mathbf{7 5}$, 116-135.

Geyer, G., Landing, E. and Heldmaier, W. 1995. Faunas and depositional environments of the Cambrian of the Moroccan Atlas regions. Beringeria, Special Issue 2, 47 119.

Gozalo, R. y Liñán, E. 1988. Los materiales hercínicos de la Cordillera Ibérica en el contexto del Macizo Ibérico. Estudios geológicos, 44, 399-404.

Gozalo, R., Liñán, E. y Álvaro, J. 1993. Bioestratigrafía del Cámbrico Medio de Villafeliche (Prov. Zaragoza, España). Revista Española de Paleontología, no extr., 49-57.

Gozalo, R., Liñán, E. and Sdzuy, K. 1996. The Lower and Middle Cambrian of Murero and Villafeliche (West Asturian-Leonese Zone. Western Iberian Chain). In: II Field Conference of the Cambrian Stage Subdivision Working Groups. International Subcommision on Cambrian Stratigraphy. Spain. 13-21 September 1996. Field Trip Guide and Abstracts (Eds. E. Liñán, J.A. Gámez Vintaned and R. Gozalo). Universidad de Zaragoza, 73-80. 
Hughes, N.C. 1994. Ontogeny, Intraspecific Variation, and Systematics of the Late Cambrian Trilobite Dikelocephalus. Smithsonian Contributions to Paleobiology, 79, 1-89.

Hughes, N.C. and Jell, P.A. 1992. A statistical/computergraphic technique for assesing variation in tectonically deformed fossils and its application to Cambrian trilobites from Kashmir. Lethaia, 25, 317-330.

Labandeira, C.C. and Hughes, N.C. 1994. Biometry of the Late Cambrian trilobite genus Dikelocephalus and its implications for trilobites systematics. Journal of Paleontology, 68, 492-517.

Landing, E. and Westrop, S.R. 1998. Cambrian faunal sequence and depositional history of Avalonian Newfoundland and New Brunswick: Field workshop. New York State Museum Bulletin, 492, 5-75.

Lecointre, G. 1926. Recherches géologiques dans la Meseta marocaine. Mémoires du Service Géologique du Maroc, 14, 1-154.

Levi-Setti, R. 1993. Trilobites. 2nd. Edition. The University of Chicago Press, Chicago, London, $342 \mathrm{pp}$.

Liñán, E. 1978. Bioestratigrafía de la Sierra de Córdoba. Tesis doctorales de la Universidad de Granada, 191, 1212.

Liñán, E. y Gozalo, R. 1986. Trilobites del Cámbrico inferior y medio de Murero (Cordillera Ibérica). Memorias del Museo Paleontológico de la Universidad de Zaragoza, 2, 1-104.

Liñán, E., Gozalo, R., Gámez, J.A. y Álvaro, J.J. 1992. Las formaciones del Grupo Mesones (Cámbrico InferiorMedio) en las Cadenas Ibéricas. III Congreso Geológico de España y VIII Congreso Latinoamericano de Geología, Salamanca, Actas, 1, 517-523.

Liñán, E., Perejón, A. and Sdzuy, K. 1993. The LowerMiddle Cambrian stages and stratotypes from the Iberian Peninsula: a revision. Geological Magazine, 130, 817833.

Liñán, E., Álvaro, J.J., Gozalo, R., Gámez-Vintaned, J.A. y Palacios, T. 1995. El Cámbrico Medio de la Sierra de Córdoba (Ossa-Morena, S de España): trilobites y paleoicnología. Implicaciones bioestratigráficas y paleoambientales. Revista Española de Paleontología, 10, 219-238.

Liñán, E., Sdzuy, K., Álvaro, J.J., Gámez Vintaned, J.A. and Gozalo, R. 1996. September 19. The Jarque section: Lower and Middle Cambrian (West Asturian-Leonese Zone. Eastern Iberian Chain). In: II Field Conference of the Cambrian Stage Subdivision Working Groups. International Subcommision on Cambrian Stratigraphy. Spain. 13-21 September 1996. Field Trip Guide and Abstracts (Eds. E. Liñán, J.A. Gámez Vintaned and R. Gozalo). Universidad de Zaragoza, 57-72.

Mansuy, H. 1923. Description de quelques Trilobites du Cambrien du Maroc. Bulletin de la Société géologique de France, ser. 4, 22, 285-289.

Martin, F. and Dean, W. T. 1988. Middle and Upper Cambrian acritarch and trilobite zonation at Manuels river and Random island, Eastern Newfoundland. Geological Survey of Canada, Bulletin, 381, 1-91.

Matthew, G. F. 1887. On the Cambrian faunas of Cape
Breton and Newfounland. Transactions of the Royal Society of Canada, section 4, 4, 147-157.

Palacios, T. 1982. El Cámbrico entre Viniegra de Abajo y Mansilla (Sierra de la Demanda, Logroño). Trilobites e icnofósiles: Biblioteca de Estudios Riojanos, Logroño, $85 \mathrm{pp}$.

Rushton, A.W.A. and Hughes, N.C. 1996. Biometry, systematics and biogeography of the late Cambrian trilobite Maladioidella abdita. Transactions of the Royal Society of Edinburgh: Earth Sciences, 86, 247-256.

Schmidt, E. W. 1942. Die mittelkambrische Fauna von Doberlug. Jahrbuch des Reichsamts für Bodenforschung, 62, 344-402.

Sdzuy, K. 1957. Revision der mittelkambrischen Trilobiten von Doberlug. Senckenbergiana lethaea, 38 (1-2), 7-28.

Sdzuy, K. 1958. Neue Trilobiten aus dem Mittelkambrium von Spanien. Senckenbergiana lethaea, 39 (1-2), 235 253.

Sdzuy, K. 1968. Trilobites del Cámbrico Medio de Asturias. Trabajos de Geología, Universidad de Oviedo, 1 (1967), 77-133.

Sdzuy, K. 1969. Bioestratigrafía de la Griotte Cámbrica de los Barrios de Luna (León) y de otras sucesiones comparables. Trabajos de Geología, Universidad de Oviedo, 2 (1968), 45-58.

Sdzuy, K., Liñán, E. and Gozalo, R. 1996. The Middle Cambrian Series. In II Field Conference of the Cambrian Stage Subdivision Working Groups. International Subcommission on Cambrian Stratigraphy. Spain, 13-21 September 1996. Field Trip Guide and Abstracts (eds. E. Liñán, J.A. Gámez Vintaned and R. Gozalo). Universidad de Zaragoza, 16-18.

Sdzuy, K., Liñán, E. and Gozalo, R. 1999. The Leonian Stage (early Middle Cambrian): a unit for Cambrian correlation in the Mediterranean subprovince. Geological Magazine, 136, 39-48.

Shimer, H.W. and Shrock, R. R. 1944. Index fossils of North America. The Massachusetts Institute of Technology Press, Cambridge, 1-837.

Skehan, J.W., Murray, D.P., Palmer, A.R., Smith, A.T. and Belt, E.S. 1978. Significance of fossiliferous Middle Cambrian rocks of Rhode Island to the history of the avalonian microcontinent. Geology, 6, 694-698.

Thoral, M. 1935. Contribution à l'étude paléontologique de l'Ordovicien inférieur de la Montagne Noire et révision sommaire de la faune cambrienne de la Montagne Noire. Imprimerie de la Charité, Montpellier, 1-362.

Thoral, M. 1947. Trois nouveaux genres des Trilobites acadiens du Languedoc et d'Espagne. Comptes Rendus de l'Académie des Sciences Paris, 224, 59-60.

Valenzuela, J.I., Gámez, J.A, Liñán, E. y Sdzuy, K. 1990. Estratigrafía de la región de Brea. Cadena Ibérica Oriental. Boletín de la Real Sociedad Española de Historia Natural (Sección Geológica), 85, 45-54.

Walcott, C.D. 1884. On the Cambrian faunas of North America. U.S. Geological Survey, Bulletin, 10, 289-355.

Webster, M. and Hughes, N.C. 1999. Compaction-related deformation in Cambrian Olenelloid Trilobites and its implications for fossil morphometry. Journal of Paleontology, 73, 355-371. 
Westrop, S.R. and Landing, E. 2000. Lower Cambrian (Branchian) trilobites and biostratigraphy of the Hanford Brook Formation, Southern New Brunswick. Journal of Paleontology, 74, 858-878.

Wheeler, R.R. 1942. New Mid-Cambrian ptychoparid.
American Journal of Science, 240, 567-570.

Zamarreño, I. 1972. Las litofacies carbonatadas del Cámbrico de la Zona Cantábrica (NW de España) y su distribución paleogeográfica. Trabajos de Geología, Universidad de Oviedo, 5, 1-118.

Manuscrito recibido: 1 de julio, 2002

Manuscrito aceptado: 7 de noviembre, 2002

\section{APÉNDICE}

Dimensiones de los caracteres cranidiales (en $\mathrm{mm}$ ) e índices de los ejemplares de Badulesia tenera utilizados en este trabajo. Sd'68: Sdzuy (1968) lámina 6. D’86: Dean et al. (1986) lámina 10. L-S’93: Levi-Setti (1993) lámina 111.

\begin{tabular}{|c|c|c|c|c|c|c|c|c|c|c|c|c|}
\hline MPZ & Le & $\mathrm{Lg}$ & Lb & Lap & Laf & Ac & Ac2 & $\mathrm{Ag}$ & Morf & Lg/Lc & Lb/Lc & $\mathrm{Ag} / \mathrm{Ac}$ \\
\hline $2001 / 131$ & 4,29 & 3.57 & 0,71 & 00 & 0,71 & 5,71 & 4,71 & 2,14 & $\mathrm{~A}$ & 0,83 & 0,17 & 0,37 \\
\hline $2001 / 132 a$ & 14 & 29 & 0,86 & 0,00 & 0,86 & 7,14 & 29 & & & 0,83 & 0,17 & 44 \\
\hline $2001 / 132 b$ & 29 & 20 & & 0,57 & 1,00 & 5,71 & 5,29 & & & 0,77 & 10 & 45 \\
\hline 2001 & 57 & 20 & 1,29 & 0,00 & 1,29 & 6,43 & 6,43 & 2,57 & & 0,77 & 23 & 40 \\
\hline 34 & 70 & 4,40 & 1,30 & 0,00 & 1,30 & 7,14 & 7,14 & 3,29 & & 0,77 & 0,23 & 46 \\
\hline 200 & 86 & 86 & 1,00 & 0,00 & 1,00 & 5,57 & 5,00 & 2,57 & & 0,79 & 0,21 & 0,46 \\
\hline 200 & 6,71 & 5,14 & 0,71 & 0,86 & 1,57 & 9,00 & 7,86 & 3,14 & & 0,77 & 0,11 & 35 \\
\hline 200 & 3,71 & 3,00 & 0,71 & 0,00 & 0,71 & 5,14 & 4,43 & 2,14 & & 0,81 & 0,19 & 72 \\
\hline 200 & 2,86 & 2,29 & 0,57 & 0,00 & 0,57 & 4,43 & 3,43 & 2,00 & & 0,80 & 0,20 & $4 J$ \\
\hline 200 & 4,00 & 3,29 & 0,71 & 0,00 & 0,71 & 5,43 & 4,86 & & & 0,82 & 0,18 & 72 \\
\hline 200 & 3,86 & 3,14 & 0.29 & 0,43 & 0,72 & & 4,57 & & & 0,81 & 0,07 & \\
\hline 200 & 29 & 1,86 & 0 & 0,00 & 0 , & & 2,43 & 1,14 & & 0,81 & 0,19 & \\
\hline 200 & 43 &, 14 & 1,2 & 0,00 & & & 7,14 & 3,14 & & 0,80 & 0,20 & 37 \\
\hline 200 & 29 & 3,30 & 1,0 & 0,00 & 1,00 & 5 , & 5,00 & 2,57 & & 0,77 & 0,23 & \\
\hline 200 & 57 & 29 & 1,2 & 0,00 & 1,29 & & & 2,71 & & 0,77 & & \\
\hline 200 & 71 & 86 & 0,2 & 0,57 & 0,8 & & 3, & 1,8 & & 0,77 & & \\
\hline 200 & 4,86 & 4,00 & 0 & 0,00 & 0 & 5 & & 2,1 & & & & \\
\hline 200 & 5,71 & 86 & & 0,00 & 0. & & & & & & & \\
\hline 200 & 5,14 & - & 1,14 & 0,00 & & & & & & & & \\
\hline 200 & 4,80 & 3,86 & $0,8 \mathrm{c}$ & 0,00 & & 7. & & & A & & & \\
\hline 200 & 4,00 & 3,29 & 0 & 0,00 & & & & & A & & & \\
\hline 200 & 2,14 & 1,71 & 0, & 0,00 & 0, & & & 0,8 & A & & & \\
\hline 200 & 5,71 & 57 & 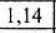 & 0,00 & & & & 3, & A & & & \\
\hline 200 & 5,71 & 71 & 1,0 & 0,00 & 1, & & & 3,0 & A & & & \\
\hline 200 & 6,43 & 14 & 43 & 0,86 & 1, & 8 & & 3 , & B & & & \\
\hline 200 & 5,1 & 4,29 & 0,8 & 0,00 & 0 & 6 , & & 2,7 & $\mathrm{~A}$ & & & \\
\hline & 5,0 & 00 & - & 0,00 & 1,0 & & & 2,7 & $\mathrm{~A}$ & & & \\
\hline 200 & 3,5 & 71 & 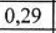 & 0,57 & 0,8 & & 4,29 & 1,8 & - & & & \\
\hline 200 & 7,0 & 71 & 20 & 0,00 & & 7, & 6,71 & 3,4 & $\mathrm{~A}$ & 2 & & \\
\hline 200 & 4,2 & 3,43 & 0 & 0,00 & 0,8 & 6,2 & 5,57 & 2,86 & A & 0,80 & & \\
\hline & 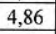 & 3,8 & & 0,00 & 1,0 & & & 2,29 & A & 9 & & \\
\hline & s & 3,5 & & 0,57 & 1,0 & 4,57 & & 1,71 & B & 78 & & 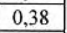 \\
\hline & 4,2 & 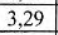 & & 0,00 & 1,0 & & 5,29 & 2,57 & $\mathrm{~A}$ & 0,77 & 3 & J \\
\hline & 4,0 & - & & 0,00 & 0,71 & & 4,86 & 2,14 & $\mathrm{~A}$ & 0,82 & & 36 \\
\hline & 5,2 & &, 00 & 0,00 & 1,00 & & 5,00 & 2,14 & $\mathrm{~A}$ & 0,81 & 19 & 37 \\
\hline & 6,4 & . & & 0,00 & 1,29 & 7,57 & 6,71 & 3,14 & $\mathrm{~A}$ & 0,80 & 20 & 42 \\
\hline & 5,43 &, 2 & 57 & 0,57 & 1,14 & 7,14 & 6,43 & 3,14 & B & 0,79 & 0,11 & 44 \\
\hline & 7,71 & 6,1 & 11 & 0,86 & 1,57 & 10,29 & 9,71 & 4,14 & B & 0,80 & 09 &, 40 \\
\hline & 4,00 & 3,00 & 00 & 0,00 & 1,00 & 5,71 & 5,00 & 2,29 & $\mathrm{~A}$ & 0,75 & 25 & 40 \\
\hline & 3,5 & 3,00 & 0,57 & 0,00 & 0,57 & 5,29 & 4,14 & 1,86 & A & 0,84 & 16 & 35 \\
\hline & 6,29 & 5,00 & 1,29 & 0,00 & 1,29 & 9,14 & 7,14 & 3,29 & $\mathrm{~A}$ & 0,80 & 20 & 36 \\
\hline $2001 / 169$ & 4,29 & 3,14 & 0,29 & 0,57 & 0,86 & 3,43 & 3,14 & 1,14 & B & 0,73 & 0,07 & 0,53 \\
\hline $2001 / 170$ & 4,29 & 3,57 &, 29 & 0,43 & 0,71 & 5,00 & 4,00 & 2,00 & $\mathrm{~B}$ & 0,83 & 0,07 & 0,40 \\
\hline
\end{tabular}

\begin{tabular}{|c|c|c|c|c|c|c|c|c|c|c|c|c|}
\hline MPZ & Lc & $\mathrm{Lg}$ & Lb & Lap & Laf & Ac & & $\cdots$ & 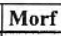 & $\mathrm{Lg} / \mathrm{Lc}$ & Lb/Lc & \\
\hline $2001 / 172$ & 6,29 & 5,14 & 0,43 & 0,71 & 1,14 & 8,57 & 6,57 & & B & 0,82 & 0,07 & \\
\hline $2001 / 173$ & 3,57 & 2,71 & 8 &, 00 & 0,86 & & & & & 76 & & \\
\hline 2001 & 2,86 & 2,14 & 0,71 &, 00 &, 71 & & 3,86 & 1,71 & & 75 & & \\
\hline & 3,71 & 2,57 & 0,57 & 0,57 & 1,14 & 6,00 & 5,29 & $=$ & &, 69 & & \\
\hline & 4,00 & 3,00 & 0,43 & 0,57 & 1,00 & & 2,43 & 1,29 & &, 75 & & \\
\hline & 3,57 & 2,57 & 0,43 & 0,57 & 1,00 & & & 1,8 & &, 72 & & \\
\hline & 4,86 & 3,86 & 1,00 & 0,00 & 1,00 & & 5,00 & 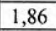 & &, 79 & & \\
\hline & 3,43 & 2,71 & 0,71 &, 00 &, 71 & & & - & &, 79 & & \\
\hline & 5,00 & 3,57 & 0,71 &, 71 & 1,43 & & 6,71 & - & & 0,71 & & \\
\hline & 5,29 & 4,00 & 1,29 & 0,00 & & & 5,71 & 2 , & &, 76 & & \\
\hline & 7,43 & 6,00 & 0,57 & 0,86 & & & 7,14 & 3, & & 8,81 & & \\
\hline & 2,71 & 2,14 & 0,57 & 0,00 & & & 3,00 & 1,2 & &, 79 & & \\
\hline & 3,43 & 2,71 & 0,7 & 0,00 & & & 5,00 & 2, & & 79 & & \\
\hline & 3,43 & 2,71 & 0 , & 0,00 & & & 4,71 & & &, 79 & & \\
\hline & 571 & 4,57 & 1 , & 00 & & & 5, & & & 80 & & \\
\hline & 5,00 & 3,71 & 0, & , & & & 4,86 & 2 , & & 74 & & \\
\hline & 420 & 3,14 & 1, & 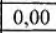 & & & 5 , & 2 , & &, 73 & & \\
\hline & 471 & 3,86 & 0, & 00 & & & 4, & 2 , & & 82 & & \\
\hline & 500 & 4,10 & 0 , & & & & 5 , & 2 , & & & & \\
\hline & 6,00 & 4,50 & 1, & 00 & & & 8 & 3, & & 75 & & \\
\hline & 8 & 8,00 & 2 , & & & & 10,00 & 4, & & 77 & & \\
\hline & 414 & 3,00 & 0 & $v_{0}$ & & & 3,57 & 1 & & & & \\
\hline & 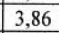 & 3,00 & 0 & & & & 4 , & 2, & & &, 11 & \\
\hline & 4,40 & 3,40 & 1,0 & 0 & 1, & & 4, & 2 & & 77 & 2 & \\
\hline & 6,00 & 4,57 & 1, & 0 & 1 & & 6 , & 3 & & 76 &, 24 & \\
\hline & & 3,00 & 1, & & & & 4 , & 2 , & & & 5 & \\
\hline & 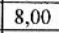 & 6,57 & & & 1 & & 8 & 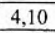 & & &, 09 & \\
\hline & 5,70 & 4,20 & 1, & & 1, & & 7, & 3 , & & & & \\
\hline & 3,40 & 2,70 & 0, & & 0 , & 4, & 3 & 1 & & 79 & 21 & \\
\hline & 4,20 & 3,20 & 1,0 & & 1,0 & 5,80 & 5 , & 2 & & & & \\
\hline & 5,40 & 4,10 & 1,3 & & 1,3 & 7,00 & 6 , & & & & & \\
\hline & & 6,00 & 2,0 & & 2,00 & 11,71 & 12,00 & & & & & \\
\hline & & 4,00 & 0,7 & & 1,33 & & & & & &, 13 & \\
\hline & 7,20 & 5,70 & 1,5 & & 1,50 & 10,50 & 10,00 & & & & & \\
\hline & 4,00 & 3,10 & & & & & & & & & & \\
\hline & 11,00 & 8,67 & & & 2,33 & 16,67 & 15,00 & & & & & \\
\hline & 7,50 & 5,80 & 1,7 & & 1,70 & 0 & & 3 & & & & \\
\hline \begin{tabular}{|l|} 
Sd'68: 12 \\
\end{tabular} & 7,50 & 5,70 & 1,8 & & 1,80 & 11 & & & & & 24 & \\
\hline & 8,00 & 6,00 & 2,0 & & 2 , & & $\pi$ & & & & & \\
\hline & & 3,2 & 1,0 & & 1, & 5 , & 4 & 2 & & & & \\
\hline & & 3,7 & & & 0, & 6 , & & 2, & H & & & \\
\hline & & 2,40 & 0,8 & & 0,80 & 4, & $J$, & 1, & & & & \\
\hline L-S'93 & 7,23 & 5,39 & 1,84 & 0,00 & 1,84 & 11,00 & 9,00 & 4,00 & A & 0,75 & 0,25 & 0,3 \\
\hline
\end{tabular}

